\title{
RANKS OF COMMUTATORS AND GENERALIZED SEMICOMMUTATORS OF QUASIHOMOGENEOUS TOEPLITZ OPERATORS
}

\author{
XING-TANG DONG AND ZE-HUA ZHOU
}

\begin{abstract}
We study the ranks of commutators and generalized semicommutators of Toeplitz operators with quasihomogeneous symbols on both the harmonic Bergman space and the Bergman Space. In particular, when one of quasihomogeneous symbols is the the form of $e^{i k \theta} r^{m}$, we first obtain specific sufficient and necessary conditions for commutators and generalized semicommutators to be finite rank. Then we make further efforts to determine the range of each finite rank commutator and generalized semicommutators, and consequently the explicit canonical form and the rank are obtained. Thus, the finite rank problem of commutators and generalized semicommutators of such special Toeplitz operators is completely solved. As applications, several interesting corollaries and nontrivial examples are given. Also, we show close connections of the finite rank problem between the harmonic Bergman space and Bergman space cases.
\end{abstract}

\section{INTRODUCTION}

Let $d A$ denote the Lebesgue area measure on the unit disk $D$, normalized so that the measure of $D$ equals 1 . The harmonic Bergman space $L_{h}^{2}$ is the closed subspace of $L^{2}(D, d A)$ consisting of the harmonic functions on $D$. Given $z \in D$, let $K_{z}(w)=\frac{1}{(1-w \bar{z})^{2}}$ be the well-known reproducing kernel for the Bergman space $L_{a}^{2}$ consisting of all $L^{2}$-analytic functions on $D$. Since $L_{h}^{2}=L_{a}^{2}+\overline{L_{a}^{2}}$, it is easily checked that the reproducing kernel $R_{z}$ in $L_{h}^{2}$ is

$$
R_{z}=K_{z}+\overline{K_{z}}-1 \text {. }
$$

Thus, each $R_{z}$ is real-valued and the orthogonal projection $Q$ from $L^{2}(D, d A)$ onto $L_{h}^{2}$ can be represented by

$$
Q f(z)=\int_{D}\left(\frac{1}{(1-z \bar{w})^{2}}+\frac{1}{(1-\bar{z} w)^{2}}-1\right) f(w) d A(w)
$$

for $f \in L^{2}(D, d A)$. Recall the well-known Bergman projection $P$ has the following integral formula

$$
P f(z)=\int_{D} f(w) \overline{K_{z}(w)} d A(w),
$$

and hence $Q$ can be rewritten as $Q f=P f+\overline{P \bar{f}}-P f(0)$.

For $u \in L^{1}(D, d A)$, we define an operator $T_{u}$ with symbol $u$ on $L_{h}^{2}$ by

$$
T_{u} h=Q(u h)
$$

for $h \in L_{h}^{2}$. This operator is always densely defined on the polynomials and not bounded in general. We are interested in the case where this densely defined operator is bounded in the $L_{h}^{2}$ norm. In this paper we will consider the case $u$ is a T-function, following [25].

Definition 1.1. Let $F \in L^{1}(D, d A)$.

(a) We say that $F$ is a T-function if the equation (1.1), with $u=F$, defines a bounded operator on $L_{h}^{2}$.

(b) If $F$ is a T-function, we write $T_{F}$ for the continuous extension of the operator defined by (1.1). We say that $T_{F}$ is a Toeplitz operator if and only if $T_{F}$ is defined in this way.

2010 Mathematics Subject Classification. Primary 47B35; Secondary 47B47.

Key words and phrases. quasihomogeneous Toeplitz operators, harmonic Bergman space, Bergman space, finite rank, commutator, generalized semicommutator. 
(c) If there is an $r \in(0,1)$ such that $F$ is (essentially) bounded on the annulus $\{z: r<|z|<1\}$, then we say that $F$ is "nearly bounded".

Generally, the T-functions form a proper subset of $L^{1}(D, d A)$ which contains all bounded and "nearly bounded" functions.

A function $f$ is said to be quasihomogeneous of degree $k \in \mathbb{Z}$ if

$$
f\left(r e^{i \theta}\right)=e^{i k \theta} \varphi(r),
$$

where $\varphi$ is a radial function. In this case the associated Toeplitz operator $T_{f}$ is also called quasihomogeneous Toeplitz operator of degree $k$. By a straightforward deduction, one can see that $e^{i k \theta} \varphi(r)$ is a T-function if and only if $\varphi(r)$ is a T-function.

For two Toeplitz operators $T_{f_{1}}$ and $T_{f_{2}}$, we define the commutator and the semicommutator respectively by

and

$$
\left[T_{f_{1}}, T_{f_{2}}\right]=T_{f_{1}} T_{f_{2}}-T_{f_{2}} T_{f_{1}}
$$

$$
\left(T_{f_{1}}, T_{f_{2}}\right]=T_{f_{1}} T_{f_{2}}-T_{f_{1} f_{2}} .
$$

For another Toeplitz operator $T_{f}$, the generalized semicommutator is defined by $T_{f_{1}} T_{f_{2}}-T_{f}$.

A major goal in the theory of Toeplitz operators on the function spaces is to describe the zero commutator or generalized semicommutator of given Toeplitz operators. The corresponding problems on the classical Hardy space were completely solved by Brown and Halmos in [5]. However, in the setting of the Bergman space (or the harmonic Bergman space), only some special Toeplitz operators were considered. For example, harmonic Toeplitz operators has been investigated in 1, 2, 10, 11, 12, 31 at an earlier time, and recently there has been an increasing interest in quasihomogeneous Toeplitz operators, see, e.g. 66, 7, 8, 16, 18, 19, 20, 21, 22, 25, 26, 27, 28, 29, 30, 32. The natural question to ask is why the quasihomogeneous Toeplitz operator is becoming the focus in this field. Perhaps there are mainly three reasons for it:

(1) It was shown in [16] that any function $f \in L^{2}(D, d A)$ has the following polar decomposition: $f\left(r e^{i \theta}\right)=\sum_{k \in \mathbb{Z}} e^{i k \theta} f_{k}(r)$. Obviously, each element of the polar decomposition is quasihomogeneous. So in order to better understand and research general Toeplitz operators, a more complete study of quasihomogeneous Toeplitz operators is needed.

(2) Quasihomogeneous function can be regarded as the generalization of monomial $z^{m} \bar{z}^{n}$. As is generally known, monomial Toeplitz operator, particularly when the symbol is analytic (or co-analytic) monomial, plays an important role in exploring basic algebraic properties of Toeplitz operators. Therefore, the research of quasihomogeneous Toeplitz operators in itself is quite meaningful.

(3) There are many nontrivial and complex results about quasihomogeneous Toeplitz operators. For example, if thinking of analytic functions being placed on the real axis, conjugate analytic on the imaginary axis and the radial functions on the diagonal $y=x$ in the first quadrant, then it was showed in [16 that there will be many lines parallel to the diagonal, "holding" a symbol that gives a Toeplitz operator commuting with $T_{z^{m}} \bar{z}^{n}$ on $L_{a}^{2}$. Moreover, each of these lines will hold no more than one such symbol.

In this paper, we continue to further study the property of the commutator or generalized semicommutator of given Toeplitz operators, i.e., finite rank problem. Recall that an operator $A$ on a Hilbert space $H$ is said to have finite rank if the closure of $\operatorname{Ran}(A)$ which is the range of the operator has finite dimension. For a bounded finite rank operator $A$ on $H$, define $\operatorname{rank}(A)=\operatorname{dim} \operatorname{Ran}(A)$.

On the Hardy space of the unit disk, the problem of determining when the commutator or semicommutator has finite rank has been completely solved in [4, 17]. Also, the same problem has been studied on $L_{a}^{2}$. Guo, Sun and Zheng [23] showed that there is no nonzero finite rank commutator or semicommutator of harmonic Toeplitz operators. Then Čučković 13 ] studied the finite rank perturbation of the Brown-Halmos type results involving products of harmonic Toeplitz operators, which is called finite rank problem of generalized semicommutator in this paper. Unlike the harmonic symbol case, Čučković and Louhichi [15] later showed that 
commutators and semicommutators of two quasihomogeneous Toeplitz operators of opposite degrees can be nonzero finite rank operators. More recently, Čučković and Le [14 obtained results on finite rank commutators and semicommutators of harmonic Toeplitz operators on Bergman space of polyanalytic functions on the unit disk. On the contrast to the Hardy space and Bergman space cases, the conditions on harmonic Bergman space are quite different. Chen, Koo and Lee [9] showed that the commutator of any two Toeplitz operators with general symbols can't have an odd rank.

Obviously, only a few results of finite rank problem were known on both $L_{a}^{2}$ and $L_{h}^{2}$, and many seemingly simple and yet very complicated problems are still open. Take the commutator of monomial Toeplitz operators for example: When the commutator $\left[T_{e^{i k_{1} \theta} r^{m_{1}}}, T_{e^{i k_{2} \theta} r^{m_{2}}}\right]$ on $L_{a}^{2}$ (or on $L_{h}^{2}$ ) has finite rank remain to be solved. As one of interesting applications of our main theorem, we will give a complete characterization for it, including the range, the rank and the explicit canonical form of each finite rank commutator.

The paper is organized as follows. Motivated by [9], we first give some quite unexpected results in Section 2: The generalized semicommutator $T_{f_{1}} T_{f_{2}}-T_{f}$ with general symbols on $L_{h}^{2}$ has finite rank if and only if the anti-ordered generalized semicommutator $T_{f_{2}} T_{f_{1}}-T_{f}$ has finite rank. As a consequence, the finite rank generalized semicommutator $T_{f_{1}} T_{f_{2}}-T_{f}$ on $L_{h}^{2}$ implies that the commutator $\left[T_{f_{1}}, T_{f_{2}}\right]$ must be finite rank. Obviously, this high correlation will be especially useful when we study the rank of the generalized semicommutator.

Returning to the quasihomogeneous symbol case, Section 3 contains some lemmas which will be used later. In Sections 4, we discuss finite rank commutators of quasihomogeneous Toeplitz operators on $L_{h}^{2}$. When one of symbols is the the form of $e^{i k \theta} r^{m}$ with $(k, m) \in \mathbb{Z} \times[-1,+\infty)$, the results have some aspects we wish to emphasize. Our results involve not only several specific necessary and sufficient conditions for commutators to be finite rank, but also the range, the rank and the explicit canonical form of the finite rank commutator in each condition. Much effort has gone into determining the range of each finite rank commutator. In fact, we first get the most likely range of finite rank commutator $\left[T_{e^{i k_{1} \theta} r^{m}}, T_{e^{i k_{2} \theta} \varphi}\right]$. Then using the symmetry number of the commutator together masterly handling of the coefficient with the monotonicity of functions appeared in Lemma 3.5 and Lemma 3.6, we verify that it is accurate.

As applications, we first give four different kinds of examples corresponding to the nonzero rank conditions of the main theorem. Then the finite rank commutator $\left[T_{e^{i k_{1} \theta} r^{k_{1}}}, T_{e^{i k_{2} \theta} \varphi}\right]$ is considered, one of whose symbols is analytic or co-analytic. Also, the corresponding problem of the commutator $\left[T_{e^{i k_{1} \theta} r^{m_{1}}}, T_{e^{i k_{2} \theta} r^{m_{2}}}\right]$ is studied.

If all similar questions are raised under the condition of generalized semicommutators on $L_{h}^{2}$, we can also obtain the complete descriptions in Section 5. In fact, using the results in Section 2 and in Section 4, it is relatively easy to get necessary conditions for generalized semicommutators to be finite rank, but a further analysis is needed to get corresponding results of generalized semicommutators, particularly to determine the range of each finite generalized semicommutator. Of cause, the finite rank problem of the semicommutator $\left(T_{e^{i k_{1} \theta_{r}}}, T_{e^{i k_{2} \theta} \varphi}\right]$, as an application of main theorem, is completely solved in this section.

In Section 6 we modify our arguments used in previous sections to obtain corresponding results on the Bergman space. To end this paper we will show some connections between finite rank commutators (or generalized semicommutators) of quasihomogeneous Toeplitz operators on the Bergman space and on the harmonic Bergman space.

\section{Rank equivalence of generalized semicommutators of Toeplitz Operators}

When dealing with the finite rank operators, the following notation is advantageous. For two nontrivial functions $\phi$ and $\psi$ in $L_{h}^{2}$, define the operator $\phi \otimes \psi$ of rank one to be

$$
(\phi \otimes \psi) h=\langle h, \psi\rangle \phi, \quad h \in L_{h}^{2} .
$$

We list here some well known and easy properties of $\phi \otimes \psi$, which will be used later.

Proposition 2.1. For two nontrivial functions $\phi$ and $\psi$ in $L_{h}^{2}$, the following statements hold. 
(a) $\operatorname{Ran}(\phi \otimes \psi)=\mathbb{C} \phi$ and $\operatorname{ker}(\phi \otimes \psi)=[\psi]^{\perp}$.

(b) $[\phi \otimes \psi]^{*}=\psi \otimes \phi$. Here $[\phi \otimes \psi]^{*}$ denotes the adjoint operator of $\phi \otimes \psi$.

(c) $\overline{(\phi \otimes \psi)(h)}=(\bar{\phi} \otimes \bar{\psi})(\bar{h})$.

(d) Every operator of finite rank $N \geq 1$ on $L_{h}^{2}$ has a canonical form: $\sum_{j=1}^{N} \phi_{j} \otimes \psi_{j}$ for some linearly independent sets $\left\{\phi_{1}, \cdots, \phi_{N}\right\}$ and $\left\{\psi_{1}, \cdots, \psi_{N}\right\}$ in $L_{h}^{2}$.

We are now ready to state and prove our result about rank equivalence of generalized semicommutators of Toeplitz operators with general symbols.

Theorem 2.2. Let $f_{1}, f_{2}$ and $f$ be T-functions on $D$. If the generalized semicommutator $T_{f_{1}} T_{f_{2}}-T_{f}$ has finite rank $N$ on $L_{h}^{2}$, then the anti-ordered generalized semicommutator $T_{f_{2}} T_{f_{1}}-$ $T_{f}$ also has finite rank $N$.

Proof. Since $R_{a}$ is real-valued for each $a \in D$, we note $\overline{Q(g)}=Q(\bar{g})$ for every $g \in L^{2}(D, d A)$, and hence

$$
\begin{aligned}
\overline{\left(T_{f_{2}} T_{f_{1}}-T_{f}\right)(h)} & =Q\left(\overline{f_{2}} Q\left(\overline{f_{1} h}\right)\right)-Q(\overline{f h}) \\
& =\left(T_{\overline{f_{2}}} T_{\overline{f_{1}}}-T_{\bar{f}}\right)(\bar{h})=\left(T_{f_{1}} T_{f_{2}}-T_{f}\right)^{*}(\bar{h})
\end{aligned}
$$

for any $h \in L_{h}^{2}$. Hence $\left(T_{f_{2}} T_{f_{1}}-T_{f}\right)(h)=\overline{\left(T_{f_{1}} T_{f_{2}}-T_{f}\right)^{*}(\bar{h})}$.

Suppose $T_{f_{1}} T_{f_{2}}-T_{f}$ has finite rank $N$. If $N=0$, then it follows that $T_{f_{2}} T_{f_{1}}-T_{f}=0$, as desired. So assume $N \geq 1$. We write

$$
T_{f_{1}} T_{f_{2}}-T_{f}=\sum_{j=1}^{N} \phi_{j} \otimes \psi_{j}
$$

for some linearly independent sets $\left\{\phi_{1}, \cdots, \phi_{N}\right\}$ and $\left\{\psi_{1}, \cdots, \psi_{N}\right\}$ in $L_{h}^{2}$. Then by Proposition 2.1, we get

$$
\left(T_{f_{2}} T_{f_{1}}-T_{f}\right)(h)=\sum_{j=1}^{N} \overline{\left[\phi_{j} \otimes \psi_{j}\right]^{*}(\bar{h})}=\sum_{j=1}^{N}\left[\overline{\psi_{j}} \otimes \overline{\phi_{j}}\right](h),
$$

which implies

$$
T_{f_{2}} T_{f_{1}}-T_{f}=\sum_{j=1}^{N} \overline{\psi_{j}} \otimes \overline{\phi_{j}} .
$$

So $T_{f_{2}} T_{f_{1}}-T_{f}$ also has finite rank $N$, as desired.

Theorem 2.2 yields the following interesting result, showing the connection of finite rank commutator and corresponding generalized semicommutator of Toeplitz operators on $L_{h}^{2}$.

Corollary 2.3. Let $f_{1}, f_{2}$ and $f$ be T-functions on $D$. If the generalized semicommutator $T_{f_{1}} T_{f_{2}}-T_{f}$ has finite rank $N$ on $L_{h}^{2}$, then the commutator $\left[T_{f_{1}}, T_{f_{2}}\right]$ also has finite rank, and the rank is less than or equal to $2 N$.

Proof. It follows from (2.1) and (2.2) that

$$
\left[T_{f_{1}}, T_{f_{2}}\right]=\sum_{j=1}^{N}\left[\phi_{j} \otimes \psi_{j}-\overline{\psi_{j}} \otimes \overline{\phi_{j}}\right]
$$

for some linearly independent sets $\left\{\phi_{1}, \cdots, \phi_{N}\right\}$ and $\left\{\psi_{1}, \cdots, \psi_{N}\right\}$ in $L_{h}^{2}$. Observe that the set $\left\{\phi_{1}, \cdots, \phi_{N}, \overline{\psi_{1}}, \cdots, \overline{\psi_{N}}\right\}$ may be linearly dependent, and the result follows.

As a direct consequence of Theorem 2.2 and Corollary 2.3, one can get the following interesting corollary. In fact, a particular case of it when both $f_{1}$ and $f_{2}$ are quasihomogeneous has been proved in 21, using quite different methods from those that we use here.

Corollary 2.4. Let $f_{1}$ and $f_{2}$ be two T-functions on $D$. If there exists a T-function $f$ such that $T_{f_{1}} T_{f_{2}}=T_{f}$ on $L_{h}^{2}$, then $T_{f_{2}} T_{f_{1}}=T_{f}$, and hence $T_{f_{1}} T_{f_{2}}=T_{f_{2}} T_{f_{1}}$. 


\section{Preliminary Results}

We start this section with the concept of the Mellin transform. For a radial function $f$ on $D$, the Mellin transform of $f$ is the function $\widehat{f}$ defined by

$$
\widehat{f}(z)=\int_{0}^{1} f(r) r^{z-1} d r .
$$

If $f \in L^{1}([0,1], r d r)$, then $\widehat{f}$ is well defined on the right half-plane $\{z: \operatorname{Re} z \geq 2\}$ and analytic on $\{z: \operatorname{Re} z>2\}$. For another radial function $g \in L^{1}([0,1], r d r)$, then their Mellin convolution is defined by

$$
\left(f *_{M} g\right)(r)=\int_{r}^{1} f\left(\frac{r}{t}\right) g(t) \frac{d t}{t}, 0 \leq r<1 .
$$

The Mellin convolution theorem states that $\left(\widehat{f *_{M} g}\right)(z)=\widehat{f}(z) \widehat{g}(z)$. Furthermore, it is known that $f *_{M} g$ is also in $L^{1}([0,1], r d r)$.

We shall often use the following lemma which is taken from [19, Lemma 2.1].

Lemma 3.1. Let $k \in \mathbb{Z}$ and let $\varphi$ be a radial $T$-function. Then on $L_{h}^{2}$, for each $n \in \mathbb{N}$ we have

$$
\begin{aligned}
& T_{e^{i k \theta} \varphi}\left(z^{n}\right)= \begin{cases}2(n+k+1) \widehat{\varphi}(2 n+k+2) z^{n+k}, & \text { if } n \geq-k, \\
2(-n-k+1) \widehat{\varphi}(-k+2) \bar{z}^{-n-k}, & \text { if } n<-k ;\end{cases} \\
& T_{e^{i k \theta} \varphi}\left(\bar{z}^{n}\right)= \begin{cases}2(n-k+1) \widehat{\varphi}(2 n-k+2) \bar{z}^{n-k}, & \text { if } n \geq k, \\
2(k-n+1) \widehat{\varphi}(k+2) z^{k-n}, & \text { if } n<k .\end{cases}
\end{aligned}
$$

A direct calculation gives the following essential lemma.

Lemma 3.2. Let $k, l \in \mathbb{Z}$ and let $\varphi$ be a radial $T$-function. Then on $L_{h}^{2}$,

$$
T_{e^{i k \theta} \varphi}\left(r^{|l|} e^{i l \theta}\right)=\lambda_{k, l} r^{|k+l|} e^{i(k+l) \theta}
$$

for some constant $\lambda_{k, l}$. Moreover,

$$
(|-l|+1) \lambda_{k, l}=(|l+k|+1) \lambda_{k,-l-k} .
$$

Proof. By Lemma 3.1, it can easily be checked that $T_{e^{i k \theta} \varphi}\left(r^{|l|} e^{i l \theta}\right)=\lambda_{k, l} r^{|k+l|} e^{i(k+l) \theta}$, where

$$
\lambda_{k, l}= \begin{cases}2(l+k+1) \widehat{\varphi}(2 l+k+2), & \text { if } l \geq 0 \text { and } l \geq-k \\ 2(-l-k+1) \widehat{\varphi}(-k+2), & \text { if } l \geq 0 \text { and } l \leq-k \\ 2(-l-k+1) \widehat{\varphi}(-2 l-k+2), & \text { if } l \leq 0 \text { and }-l \geq k \\ 2(l+k+1) \hat{\varphi}(k+2), & \text { if } l \leq 0 \text { and }-l \leq k\end{cases}
$$

Observe that

- $l \geq 0$ and $l \geq-k \Longleftrightarrow-l-k \leq 0$ and $l+k \geq k$;

- $l \geq 0$ and $l \leq-k \Longleftrightarrow-l-k \geq 0$ and $-l-k \leq-k$;

- $l \leq 0$ and $-l \geq k \Longleftrightarrow-l-k \geq 0$ and $-l-k \geq-k$;

- $l \leq 0$ and $-l \leq k \Longleftrightarrow-l-k \leq 0$ and $l+k \leq k$.

Then by (3.2), we get

$$
\lambda_{k,-l-k}= \begin{cases}2(l+1) \hat{\varphi}(2 l+k+2), & \text { if } l \geq 0 \text { and } l \geq-k \\ 2(l+1) \hat{\varphi}(-k+2), & \text { if } l \geq 0 \text { and } l \leq-k \\ 2(-l+1) \widehat{\varphi}(-2 l-k+2), & \text { if } l \leq 0 \text { and }-l \geq k \\ 2(-l+1) \hat{\varphi}(k+2), & \text { if } l \leq 0 \text { and }-l \leq k\end{cases}
$$

Comparing (3.2) with (3.3), it is clear that (3.1) holds. This completes the proof.

By complex and lengthy calculations, we proved the following technical lemma in [20. Using the critical equation (3.1), now we can give a new and quite simple proof. 
Lemma 3.3. Let $k_{1}, k_{2} \in \mathbb{Z}$, and let $\varphi_{1}, \varphi_{2}$ be two radial $T$-functions. Then on $L_{h}^{2}$, for any $k \in \mathbb{Z}$ we have

$$
\begin{aligned}
& T_{e^{i k_{1} \theta} \varphi_{1}} T_{e^{i k_{2} \theta} \varphi_{2}}\left(r^{|k|} e^{i k \theta}\right)=T_{e^{i k_{2} \theta} \varphi_{2}} T_{e^{i k_{1} \theta} \varphi_{1}}\left(r^{|k|} e^{i k \theta}\right) \Longleftrightarrow \\
& T_{e^{i k_{1} \theta} \varphi_{1}} T_{e^{i k_{2} \theta} \varphi_{2}}\left(r^{\left|-k-k_{1}-k_{2}\right|} e^{i\left(-k-k_{1}-k_{2}\right) \theta}\right)=T_{e^{i k_{2} \theta} \varphi_{2}} T_{e^{i k_{1} \theta} \varphi_{1}}\left(r^{\left|-k-k_{1}-k_{2}\right|} e^{i\left(-k-k_{1}-k_{2}\right) \theta}\right) .
\end{aligned}
$$

Moreover, if $-k_{1}-k_{2}$ is even, then

$$
T_{e^{i k_{1} \theta} \varphi_{1}} T_{e^{i k_{2} \theta} \varphi_{2}}\left(r^{\left|\frac{-k_{1}-k_{2}}{2}\right|} e^{\frac{i\left(-k_{1}-k_{2}\right) \theta}{2}}\right) \equiv T_{e^{i k_{2} \theta} \varphi_{2}} T_{e^{i k_{1} \theta} \varphi_{1}}\left(r^{\left|\frac{-k_{1}-k_{2}}{2}\right|} e^{\frac{i\left(-k_{1}-k_{2}\right) \theta}{2}}\right) .
$$

Proof. It follows from Lemma 3.2 that

$$
\begin{aligned}
& T_{e^{i k_{1} \theta} \varphi_{1}} T_{e^{i k_{2} \theta} \varphi_{2}}\left(r^{|k|} e^{i k \theta}\right)=T_{e^{i k_{2} \theta} \varphi_{2}} T_{e^{i k_{1} \theta} \varphi_{1}}\left(r^{|k|} e^{i k \theta}\right) \Longleftrightarrow \lambda_{k_{2}, k} \lambda_{k_{1}, k+k_{2}}=\lambda_{k_{1}, k} \lambda_{k_{2}, k+k_{1}} \\
& \Longleftrightarrow \frac{(|-k|+1) \lambda_{k_{2}, k}\left(\left|-k-k_{2}\right|+1\right) \lambda_{k_{1}, k+k_{2}}}{\left|-k-k_{2}\right|+1}=\frac{(|-k|+1) \lambda_{k_{1}, k}\left(\left|-k-k_{1}\right|+1\right) \lambda_{k_{2}, k+k_{1}}}{\left|-k-k_{1}\right|+1} .
\end{aligned}
$$

By (3.1), a direct calculation shows that the above equation is equivalent to

$$
\lambda_{k_{2},-k-k_{1}-k_{2}} \lambda_{k_{1},-k-k_{1}}=\lambda_{k_{1},-k-k_{1}-k_{2}} \lambda_{k_{2},-k-k_{2}} .
$$

Using Lemma 3.2 again, then the desired result follows immediately. Moreover, if $k_{1}-k_{2}$ is even, then using (3.1) again, we get

$$
\begin{aligned}
\lambda_{k_{2}, \frac{-k_{1}-k_{2}}{2}} \lambda_{k_{1}, \frac{-k_{1}+k_{2}}{2}} & =\frac{\left(\left|\frac{k_{1}+k_{2}}{2}\right|+1\right) \lambda_{k_{2}, \frac{-k_{1}-k_{2}}{2}}\left(\left|\frac{k_{1}-k_{2}}{2}\right|+1\right) \lambda_{k_{1}, \frac{-k_{1}+k_{2}}{2}}}{\left(\left|\frac{k_{1}+k_{2}}{2}\right|+1\right)\left(\left|\frac{k_{1}-k_{2}}{2}\right|+1\right)} \\
& =\lambda_{k_{1}, \frac{-k_{1}-k_{2}}{2}} \lambda_{k_{2}, \frac{k_{1}-k_{2}}{2}},
\end{aligned}
$$

which completes the proof.

Recall that $\frac{-k_{1}-k_{2}}{2}$ is called the symmetry number of the commutator $\left[T_{e^{i k_{1} \theta} \varphi_{1}}, T_{e^{i k_{2} \theta} \varphi_{2}}\right]$ in [20]. Since $\left\{\sqrt{|l|+1} r^{|l|} e^{i l \theta}\right\}_{l \in \mathbb{Z}}$ is an orthonormal basis for $L_{h}^{2}$, it will greatly simplify the analysis if we use this symmetry property to determine the range of the finite rank commutator two quasihomogeneous Toeplitz operators on $L_{h}^{2}$.

Lemma 3.2 implies that $T_{e^{i k \theta} \varphi}$ acts on the elements of the orthogonal basis of $L_{h}^{2}$ as a weight shift operator. From this we can give the following lemma which shows the relation between the range and the canonical form of finite rank quasihomogeneous Toeplitz operators on $L_{h}^{2}$.

Lemma 3.4. $L$ et $T_{e^{i p_{i} \theta} \varphi_{i}}, T_{e^{i q_{i} \theta} \psi_{i}}$ be quasihomogeneous Toeplitz operators on $L_{h}^{2}$ and $p_{i}+q_{i}=k$ for any $i=1, \cdots, i_{0}$. Then

$$
\operatorname{Ran}\left(\sum_{i=1}^{i_{0}} T_{e^{i p_{i} \theta} \varphi_{i}} T_{e^{i q_{i} \theta} \psi_{i}}\right)=\operatorname{span}\left\{r^{\left|k_{(1)}\right|} e^{i k_{(1)} \theta}, r^{\left|k_{(2)}\right|} e^{i k_{(2)} \theta}, \cdots, r^{\left|k_{(N)}\right|} e^{i k_{(N)} \theta}\right\}
$$

if and only if

$$
\sum_{i=1}^{i_{0}} T_{e^{i p_{i} \theta} \varphi_{i}} T_{e^{i q_{i} \theta} \psi_{i}}=\sum_{j=1}^{N} C_{k_{(j)}}\left(r^{\left|k_{(j)}\right|} e^{i k_{(j)} \theta}\right) \otimes\left(r^{\left|k_{(j)}-k\right|} e^{i\left(k_{(j)}-k\right) \theta}\right)
$$

for some nonzero constant $C_{k_{(j)}}$.

Proof. First suppose (3.4) holds. Notice that $p_{i}+q_{i}=k$, then by Lemma 3.2, for any $l \in \mathbb{Z}$

$$
\sum_{i=1}^{i_{0}} T_{e^{i p_{i} \theta} \varphi_{i}} T_{e^{i q_{i} \theta} \psi_{i}}\left(r^{|l|} e^{i l \theta}\right)= \begin{cases}0, & \text { if } l \neq k_{(j)}-k \\ C_{k_{(j)}}^{\prime} r^{\left|k_{(j)}\right|} e^{i k_{(j)} \theta}, & \text { if } l=k_{(j)}-k\end{cases}
$$


for some nonzero constant $C_{k_{(j)}}^{\prime}$, where $j \in\{1,2, \cdots, N\}$. On the other hand,

$$
\begin{aligned}
& {\left[\sum_{j=1}^{N}\left(r^{\left|k_{(j)}\right|} e^{i k_{(j)} \theta}\right) \otimes\left(r^{\left|k_{(j)}-k\right|} e^{i\left(k_{(j)}-k\right) \theta}\right)\right]\left(r^{|l|} e^{i l \theta}\right)} \\
& =\sum_{j=1}^{N}\left\langle r^{|l|} e^{i l \theta}, r^{\left|k_{(j)}-k\right|} e^{i\left(k_{(j)}-k\right) \theta}\right\rangle r^{\left|k_{(j)}\right|} e^{i k_{(j)} \theta}= \begin{cases}0, & \text { if } l \neq k_{(j)}-k, \\
\frac{1}{\left|k_{(j)}-k\right|+1} r^{\left|k_{(j)}\right|} e^{i k_{(j)} \theta}, & \text { if } l=k_{(j)}-k .\end{cases}
\end{aligned}
$$

Thus, (3.5) holds for nonzero constant $C_{k_{(j)}}=\left(\left|k_{(j)}-k\right|+1\right) C_{k_{(j)}}^{\prime}$.

The converse implication is a direct consequence of Proposition 2.1 finishing the proof.

The following two lemmas play critical roles in next two sections. Before we state our results, we need to introduce concepts of the digamma function and the polygamma function. The digamma function $\psi(z)$ is defined as the logarithmic derivative of the gamma function $\Gamma(z)$, that is:

$$
\psi(z)=\frac{d}{d z} \log \Gamma(z)=\frac{\Gamma^{\prime}(z)}{\Gamma(z)}
$$

The derivatives $\psi^{\prime}, \psi^{\prime \prime}, \psi^{\prime \prime \prime}, \ldots$ are known as the tri-, tetra-, pentagamma functions or, generally, the polygamma functions. The polygamma function may be represented as

$$
\psi^{(m)}(z)=(-1)^{m+1} \int_{0}^{+\infty} \frac{t^{m}}{1-e^{-t}} e^{-z t} d t
$$

for $\operatorname{Re} z>0$ and $m>0$. Obviously, $\psi^{\prime \prime}(s)<0$ for $s \in(0,+\infty)$, and hence $\psi^{\prime}$ is strictly monotone decreasing on $(0,+\infty)$. We refer to [3, p.260] for the properties of these functions.

Lemma 3.5. Let $b>0$, and let

$$
F(x)=\frac{\Gamma(x) \Gamma(x+b-a)}{\Gamma(x-a) \Gamma(x+b)} .
$$

(a) If $a>0$, then $F(x)$ is strictly monotone increasing on $(a,+\infty)$.

(b) If $a<0$, then $F(x)$ is strictly monotone decreasing on $(0,+\infty)$.

Proof. First suppose $a>0$. Define

$$
g(x):=\psi(x)+\psi(x+b-a)-\psi(x-a)-\psi(x+b)
$$

for $x \in(a,+\infty)$, where $\psi$ is the digamma function.

Now, we denote $h(x)=\psi(x)-\psi(x-a)$ for $x>a$. Since $a>0$, we get

$$
h^{\prime}(x)=\psi^{\prime}(x)-\psi^{\prime}(x-a)<0,
$$

and hence $h(x)>h(x+b), \forall b>0$. Consequently, $g(x)=h(x)-h(x+b)>0$ for all $x \in(a,+\infty)$. But note that $\frac{d}{d x} \log F(x)=g(x)$. So $F(x)$ is strictly monotone increasing on $(a,+\infty)$.

The proof of (b) is similar. This completes the proof.

Lemma 3.6. Let $b>0$, and let

$$
G(x)=\frac{\Gamma(-x+a) \Gamma(-x+b+1)}{\Gamma(-x+1) \Gamma(-x+b+a)} .
$$

(a) If $a \in(0,1)$, then $G(x)$ is strictly monotone increasing on $(-\infty, a)$.

(b) If $a \in(1,+\infty)$, then $G(x)$ is strictly monotone decreasing on $(-\infty, 1)$.

Proof. First suppose $a \in(0,1)$. Define

$$
g(x):=\psi(-x+a)+\psi(-x+b+1)-\psi(-x+1)-\psi(-x+b+a)
$$

for $x \in(-\infty, a)$, where $\psi$ is the digamma function.

Fix $x$ and denote $h(y)=\psi(-x+y+1)-\psi(-x+y+a)$ for $y \geq 0$. Since $a<1$, we get

$$
h^{\prime}(y)=\psi^{\prime}(-x+y+1)-\psi^{\prime}(-x+y+a)<0,
$$


Recall that $b>0$ and therefore $h(b)<h(0)$, which implies that

$$
g(x)=h(b)-h(0)<0
$$

for all $x \in(-\infty, a)$. But note that $\frac{d}{d x} \log G(x)=-g(x)$. So $G(x)$ is strictly monotone increasing on $(-\infty, a)$.

The proof of (b) is similar. This completes the proof.

Inspired by [16, we give the following lemma, which gives the sufficient and necessary conditions for the function appeared in Section 4 to be a T-function.

Lemma 3.7. Let $k_{1}, k_{2} \in \mathbb{Z}$ such that $k_{1}>0$, and let $m \in \mathbb{R}, m \geq-1$. Assume $\varphi(r)$ is a radial function on $D$ such that

$$
\widehat{\varphi}(z)=\frac{\Gamma\left(\frac{z+k_{2}}{2 k_{1}}\right) \Gamma\left(\frac{z+m+k_{1}-k_{2}}{2 k_{1}}\right)}{\Gamma\left(\frac{z+2 k_{1}-k_{2}}{2 k_{1}}\right) \Gamma\left(\frac{z+m+k_{1}+k_{2}}{2 k_{1}}\right)} .
$$

Then $\varphi(r)$ is a T-function if and only if one of the following conditions holds:

(1) $k_{2} \leq-2$ and $m+k_{1}=0$.

(2) $-2<k_{2}<m+k_{1}+2$.

(3) $k_{2} \geq m+k_{1}+2$ and $m=(2 n+1) k_{1}$ for some $n \in \mathbb{N}$.

Proof. First assume $k_{2} \leq-2$. If $\varphi(r)$ is a T-function on $D$, then $\varphi(r) \in L^{1}(D, d A)$, and hence $\widehat{\varphi}(z)$ must be well defined on $\{z: \operatorname{Re} z \geq 2\}$. Therefore, either $\frac{2 k_{1}-2 k_{2}}{2 k_{1}}$ or $\frac{m+k_{1}}{2 k_{1}}$ is an integer. Otherwise, $-k_{2}$ must be a pole of $\widehat{\varphi}(z)$ in $\{z: \operatorname{Re} z \geq 2\}$, which leads to a contradiction.

If $\frac{2 k_{1}-2 k_{2}}{2 k_{1}}$ is an integer, then we can suppose $-k_{2}=(n+1) k_{1}$ for some $n \in \mathbb{N}$, since $k_{1}>0$ and $k_{2} \leq-2$. Thus, (3.7) becomes

$$
\begin{aligned}
\widehat{\varphi}(z) & =\frac{\Gamma\left(\frac{z+k_{2}}{2 k_{1}}\right) \Gamma\left(\frac{z+m+k_{1}+k_{2}}{2 k_{1}}+n+1\right)}{\Gamma\left(\frac{z+k_{2}}{2 k_{1}}+n+2\right) \Gamma\left(\frac{z+m+k_{1}+k_{2}}{2 k_{1}}\right)} \\
& =\frac{\frac{z+m+k_{1}+k_{2}}{2 k_{1}}\left(\frac{z+m+k_{1}+k_{2}}{2 k_{1}}+1\right) \cdots\left(\frac{z+m+k_{1}+k_{2}}{2 k_{1}}+n\right)}{\frac{z+k_{2}}{2 k_{1}}\left(\frac{z+k_{2}}{2 k_{1}}+1\right) \cdots\left(\frac{z+k_{2}}{2 k_{1}}+n+1\right)} .
\end{aligned}
$$

So $\widehat{\varphi}(z)$ is a proper fraction and can be written as a sum of partial fractions $\sum_{j=0}^{n+1} \frac{A_{j}}{z+k_{2}+2 k_{1} j}$. Since $k_{2} \leq-2$ and $\widehat{\varphi}(z)$ is well defined on $\{z: \operatorname{Re} z \geq 2\}$, it follows that $A_{0}=0$, which implies

$$
\frac{m+k_{1}+k_{2}}{2 k_{1}}+i=\frac{k_{2}}{2 k_{1}}
$$

must hold for some $i \in\{0,1, \cdots, n\}$. Since $m \geq-1$ and $k_{1}>0$, we have $m+k_{1}=0$.

If $\frac{m+k_{1}}{2 k_{1}}$ is an integer, then we can suppose $m=(2 n-1) k_{1}$ for some $n \in \mathbb{N}$. If $m+k_{1} \neq 0$, then $n \geq 1$. Thus, (3.7) becomes

$$
\widehat{\varphi}(z)=\frac{\left(\frac{z-k_{2}}{2 k_{1}}+1\right)\left(\frac{z-k_{2}}{2 k_{1}}+2\right) \cdots\left(\frac{z-k_{2}}{2 k_{1}}+n-1\right)}{\frac{z+k_{2}}{2 k_{1}}\left(\frac{z+k_{2}}{2 k_{1}}+1\right) \cdots\left(\frac{z+k_{2}}{2 k_{1}}+n-1\right)}=\sum_{j=0}^{n-1} \frac{A_{j}}{z+k_{2}+2 k_{1} j} .
$$

Notice that $\frac{-k_{2}}{2 k_{1}}+i>\frac{k_{2}}{2 k_{1}}$ for all $i \in\{0,1, \cdots, n-1\}$, so $A_{0} \neq 0$, which contradicts the fact that $\widehat{\varphi}(z)$ is well defined on $\{z: \operatorname{Re} z \geq 2\}$. Thus $m+k_{1}=0$.

Conversely, assume $m+k_{1}=0$. Then (3.7) becomes $\widehat{\varphi}(z)=\frac{\Gamma\left(\frac{z+k_{2}}{2 k_{1}}\right) \Gamma\left(\frac{z-k_{2}}{2 k_{1}}\right)}{\Gamma\left(\frac{z+2 k_{1}-k_{2}}{2 k_{1}}\right) \Gamma\left(\frac{z+k_{2}}{2 k_{1}}\right)}=\frac{2 k_{1}}{z-k_{2}}$, which yields $\varphi(r)=2 k_{1} r^{-k_{2}}$, and hence $\varphi(r)$ is a T-function.

Next, assume $-2<k_{2}<m+k_{1}+2$. Then it is easy to check that $\widehat{\varphi}(z)$ is well defined on $\{z: \operatorname{Re} z \geq 2\}$ in this particular situation. Furthermore, if $\widehat{\varphi}(z)$ is a rational function, then it is obvious that $\varphi(r)$ is a T-function. Now, we suppose $\widehat{\varphi}(z)$ is a irrational function. Then using 
the same reasoning as in the proof of [16, Theorem 4], one can show that $\varphi(r) \in L^{1}(D, d A)$ and $\varphi(r)$ is "nearly bounded", which imply $\varphi(r)$ is a T-function.

Finally, assume $k_{2} \geq m+k_{1}+2$. Suppose that $\varphi(r)$ is a T-function on $D$. Similar as before, then either $\frac{-2 k_{2}}{2 k_{1}}$ or $\frac{m-k_{1}}{2 k_{1}}$ is an integer. If $\frac{-2 k_{2}}{2 k_{1}}$ is an integer, then we can suppose $k_{2}=(n+2) k_{1}$ for some $n \in \mathbb{N}$, since $k_{2}>k_{1}>0$. Thus by a simple calculation, we obtain from (3.7) that

$$
\widehat{\varphi}(z)=\frac{\frac{z+2 k_{1}-k_{2}}{2 k_{1}}\left(\frac{z+2 k_{1}-k_{2}}{2 k_{1}}+1\right) \cdots\left(\frac{z+2 k_{1}-k_{2}}{2 k_{1}}+n\right)}{\frac{z+m+k_{1}-k_{2}}{2 k_{1}}\left(\frac{z+m+k_{1}-k_{2}}{2 k_{1}}+1\right) \cdots\left(\frac{z+m+k_{1}-k_{2}}{2 k_{1}}+n+1\right)}=\sum_{j=0}^{n+1} \frac{A_{j}}{z+m+k_{1}-k_{2}+2 k_{1} j} .
$$

Since $m+k_{1}-k_{2} \leq-2$, it follows that $A_{0}=0$, which implies

$$
\frac{2 k_{1}-k_{2}}{2 k_{1}}+i=\frac{m+k_{1}-k_{2}}{2 k_{1}}
$$

must hold for some $i \in\{0,1, \cdots, n\}$, and hence $m=(2 i+1) k_{1}$.

On the other hand, if $\frac{m-k_{1}}{2 k_{1}}$ is an integer, then we can suppose $m=(2 n+1) k_{1}$ for some integer $n \geq-1$, since $m \geq-k_{1}$. However, if $n=-1$, then $m+k_{1}=0$ and (3.7) becomes

$$
\widehat{\varphi}(z)=\frac{\Gamma\left(\frac{z-k_{2}}{2 k_{1}}\right)}{\Gamma\left(\frac{z-k_{2}}{2 k_{1}}+1\right)}=\frac{2 k_{1}}{z-k_{2}}
$$

which contradicts the fact that $\widehat{\varphi}(z)$ is well defined on $\{z: \operatorname{Re} z \geq 2\}$ since $k_{2} \geq m+k_{1}+2=2$.

Conversely, assume $m=(2 n+1) k_{1}$ for some $n \in \mathbb{N}$. Then (3.7) becomes

$$
\widehat{\varphi}(z)=\frac{\Gamma\left(\frac{z+k_{2}}{2 k_{1}}\right) \Gamma\left(\frac{z-k_{2}}{2 k_{1}}+n+1\right)}{\Gamma\left(\frac{z-k_{2}}{2 k_{1}}+1\right) \Gamma\left(\frac{z+k_{2}}{2 k_{1}}+n+1\right)}=\frac{\left(\frac{z-k_{2}}{2 k_{1}}+1\right)\left(\frac{z-k_{2}}{2 k_{1}}+2\right) \cdots\left(\frac{z-k_{2}}{2 k_{1}}+n\right)}{\frac{z+k_{2}}{2 k_{1}}\left(\frac{z+k_{2}}{2 k_{1}}+1\right) \cdots\left(\frac{z+k_{2}}{2 k_{1}}+n\right)} .
$$

Clearly, $\varphi(r)$ is a T-function. This completes the proof.

\section{RANKS OF COMMUTATORS ON $L_{h}^{2}$}

In this section, we will discuss the finite rank commutators of quasihomogeneous Toeplitz operators on $L_{h}^{2}$. Throughout the rest part of this paper, we will use the following notations for brevity. For $k_{1}, k_{2} \in \mathbb{Z}$, we denote

$$
N_{1}=\max \left\{0,-k_{1},-k_{2},-k_{1}-k_{2}\right\} .
$$

Then we write

$$
\Lambda_{1}=\left\{k \in \mathbb{Z}:-N_{1}+1 \leq k \leq N_{1}+k_{1}+k_{2}-1, k \neq \frac{k_{1}+k_{2}}{2}\right\},
$$

and

$$
\Lambda_{\frac{1}{2}}=\left\{k \in \mathbb{Z}: \frac{k_{1}+k_{2}}{2}<k \leq N_{1}+k_{1}+k_{2}-1\right\} .
$$

First we characterize the finite rank commutators of two Toeplitz operators with general quasihomogeneous symbols.

Proposition 4.1. Let $k_{1}, k_{2} \in \mathbb{Z}$, and let $\varphi_{1}$ and $\varphi_{2}$ be two radial T-functions. Then the commutator $\left[T_{e^{i k_{1} \theta} \varphi_{1}}, T_{e^{i k_{2} \theta} \varphi_{2}}\right]$ has finite rank on $L_{h}^{2}$ if and only if

$$
\begin{aligned}
& 2\left(n+k_{2}+1\right) \widehat{\varphi_{1}}\left(2 n+k_{1}+2 k_{2}+2\right) \widehat{\varphi_{2}}\left(2 n+k_{2}+2\right) \\
& =2\left(n+k_{1}+1\right) \widehat{\varphi_{1}}\left(2 n+k_{1}+2\right) \widehat{\varphi_{2}}\left(2 n+2 k_{1}+k_{2}+2\right)
\end{aligned}
$$

holds for any natural number $n \geq N_{1}$. In this case,

$$
\begin{aligned}
\operatorname{Ran}\left(\left[T_{e^{i k_{1} \theta} \varphi_{1}}, T_{e^{i k_{2} \theta} \varphi_{2}}\right]\right) & =\operatorname{span}\left\{r^{\left|k_{(1)}\right|} e^{i k_{(1)} \theta}, r^{\left|k_{(2)}\right|} e^{i k_{(2)} \theta}, \cdots, r^{\left|k_{(N)}\right|} e^{i k_{(N)} \theta},\right. \\
& \left.r^{\left|-k_{(1)}+k_{1}+k_{2}\right|} e^{i\left(-k_{(1)}+k_{1}+k_{2}\right) \theta}, \cdots, r^{\left|-k_{(N)}+k_{1}+k_{2}\right|} e^{i\left(-k_{(N)}+k_{1}+k_{2}\right) \theta}\right\}
\end{aligned}
$$


for some $k_{(1)}, \cdots, k_{(N)} \in \Lambda_{\frac{1}{2}}$, and

$$
\begin{aligned}
{\left[T_{e^{i k_{1} \theta} \varphi_{1}}, T_{e^{i k_{2} \theta} \varphi_{2}}\right]=\sum_{j=1}^{N} C_{k_{(j)}} } & {\left[\left(r^{\left|k_{(j)}\right|} e^{i k_{(j)} \theta}\right) \otimes\left(r^{\left|k_{(j)}-k_{1}-k_{2}\right|} e^{i\left(k_{(j)}-k_{1}-k_{2}\right) \theta}\right)\right.} \\
& \left.-\left(r^{\left|-k_{(j)}+k_{1}+k_{2}\right|} e^{i\left(-k_{(j)}+k_{1}+k_{2}\right) \theta}\right) \otimes\left(r^{\left|-k_{(j)}\right|} e^{-i k_{(j)} \theta}\right)\right]
\end{aligned}
$$

for some nonzero constant $C_{k_{(j)}}$. Furthermore, $\operatorname{rank}\left(\left[T_{e^{i k_{1} \theta} \varphi_{1}}, T_{e^{i k_{2} \theta} \varphi_{2}}\right]\right) \leq\left|k_{1}\right|+\left|k_{2}\right|-1$ when $k_{1}+k_{2}$ is odd, and $\operatorname{rank}\left(\left[T_{e^{i k_{1} \theta} \varphi_{1}}, T_{e^{i k_{2} \theta} \varphi_{2}}\right]\right) \leq \max \left\{0,\left|k_{1}\right|+\left|k_{2}\right|-2\right\}$ when $k_{1}+k_{2}$ is even.

Proof. For any natural number $n \geq N_{1}$, by Lemma 3.1 we get

$$
\begin{aligned}
& {\left[T_{e^{i k_{1} \theta} \varphi_{1}}, T_{e^{i k_{2} \theta} \varphi_{2}}\right]\left(z^{n}\right)} \\
& =2\left(n+k_{1}+k_{2}+1\right)\left[2\left(n+k_{2}+1\right) \widehat{\varphi_{1}}\left(2 n+k_{1}+2 k_{2}+2\right) \widehat{\varphi_{2}}\left(2 n+k_{2}+2\right)\right. \\
& \left.\quad-2\left(n+k_{1}+1\right) \widehat{\varphi_{1}}\left(2 n+k_{1}+2\right) \widehat{\varphi_{2}}\left(2 n+2 k_{1}+k_{2}+2\right)\right] z^{n+k_{1}+k_{2}} .
\end{aligned}
$$

Suppose that $\left[T_{e^{i k_{1} \theta} \varphi_{1}}, T_{e^{i k_{2} \theta} \varphi_{2}}\right]$ has finite rank. Then the set $\left\{\left[T_{e^{i k_{1} \theta} \varphi_{1}}, T_{e^{i k_{2} \theta} \varphi_{2}}\right]\left(z^{n}\right): n \geq N_{1}\right\}$ contains finite linearly independent vectors, and hence there exists some natural number $N_{0} \geq$ $N_{1}$ such that

$$
\left[T_{e^{i k_{1} \theta} \varphi_{1}}, T_{e^{i k_{2} \theta} \varphi_{2}}\right]\left(z^{n}\right)=0, \forall n \geq N_{0} .
$$

Consequently, it follows from (4.4) that (4.1) holds for any $n \geq N_{0}$. According to the basic properties of Mellin transform, (4.1) can be written as

$$
\begin{aligned}
& \left(r^{2 k_{1}+2 N_{1}}\right)\left(2 n-2 N_{1}+2\right)\left(r^{k_{1}+\widehat{2 k_{2}+2} N_{1}} \varphi_{1}\right)\left(2 n-2 N_{1}+2\right)\left(\widehat{r^{k_{2}+2 N_{1}}} \varphi_{2}\right)\left(2 n-2 N_{1}+2\right) \\
& =\left(r^{2 k_{2}+2 N_{1}}\right)\left(2 n-2 N_{1}+2\right)\left(r^{k_{1}+2 N_{1}} \varphi_{1}\right)\left(2 n-2 N_{1}+2\right)\left(r^{2 k_{1}+k_{2}+2 N_{1}} \varphi_{2}\right)\left(2 n-2 N_{1}+2\right) .
\end{aligned}
$$

Since the sequence $\left(2 n-2 N_{1}+2\right)_{n \geq N_{0}}$ is arithmetic, then as in the proof of [15, Theorem 6], we can get the above equation holds for any $n \geq N_{1}$, and hence (4.1) holds for any $n \geq N_{1}$.

Conversely, assume (4.1) holds for any $n \geq N_{1}$. Then by (4.4), we get

$$
\left[T_{e^{i k_{1} \theta} \varphi_{1}}, T_{e^{i k_{2} \theta} \varphi_{2}}\right]\left(z^{n}\right)=0, \forall n \geq N_{1} .
$$

Furthermore, it follows from Lemma 3.3 that

$$
\left[T_{e^{i k_{1} \theta} \varphi_{1}}, T_{e^{i k_{2} \theta} \varphi_{2}}\right]\left(\bar{z}^{n+k_{1}+k_{2}}\right)=0, \forall n \geq N_{1},
$$

and if $-k_{1}-k_{2}$ is even, then

$$
\left[T_{e^{i k_{1} \theta} \varphi_{1}}, T_{e^{i k_{2} \theta} \varphi_{2}}\right]\left(r^{\left|\frac{-k_{1}-k_{2}}{2}\right|} e^{\frac{i\left(-k_{1}-k_{2}\right) \theta}{2}}\right)=0 .
$$

Therefore,

$$
\begin{aligned}
& \operatorname{Ran}\left(\left[T_{e^{i k_{1} \theta} \varphi_{1}}, T_{e^{i k_{2} \theta} \varphi_{2}}\right]\right) \\
& =\operatorname{span}\left\{\left[T_{e^{i k_{1} \theta} \varphi_{1}}, T_{e^{i k_{2} \theta} \varphi_{2}}\right]\left(r^{|l|} e^{i l \theta}\right): l \in \mathbb{Z},-N_{1}-k_{1}-k_{2}+1 \leq l \leq N_{1}-1, l \neq \frac{-k_{1}-k_{2}}{2}\right\} .
\end{aligned}
$$

Now, using Lemma 3.2 and the definition of $\Lambda_{1}$, it yields

$$
\operatorname{Ran}\left(\left[T_{e^{i k_{1} \theta} \varphi_{1}}, T_{e^{i k_{2} \theta} \varphi_{2}}\right]\right) \subseteq \operatorname{span}\left\{r^{|k|} e^{i k \theta}: k \in \Lambda_{1}\right\},
$$

which shows that the commutator $\left[T_{e^{i k_{1} \theta} \varphi_{1}}, T_{e^{i k_{2} \theta} \varphi_{2}}\right]$ has finite rank. Moreover, it follows from Lemma 3.3 that, if $r^{\left|k_{(j)}\right|} e^{i k_{(j)} \theta} \in \operatorname{Ran}\left(\left[T_{e^{i k_{1} \theta} \varphi_{1}}, T_{e^{i k_{2} \theta} \varphi_{2}}\right]\right)$ for some $k_{(j)} \in \Lambda_{1}$, then

$$
r^{\left|-k_{(j)}+k_{1}+k_{2}\right|} e^{i\left(-k_{(j)}+k_{1}+k_{2}\right) \theta} \in \operatorname{Ran}\left(\left[T_{e^{i k_{1} \theta} \varphi_{1}}, T_{e^{i k_{2} \theta} \varphi_{2}}\right]\right) .
$$

Therefore, the monomials in $\operatorname{Ran}\left(\left[T_{e^{i k_{1} \theta} \varphi_{1}}, T_{e^{i k_{2} \theta} \varphi_{2}}\right]\right)$ must always appear in pairs. Notice that the set $\Lambda_{1}$ is also symmetric, and hence (4.2) must hold for some $k_{(1)}, \cdots, k_{(N)} \in \Lambda_{\frac{1}{2}}$. 
Next, we want to determine the canonical form of the finite rank commutator $\left[T_{e^{i k_{1} \theta} \varphi_{1}}, T_{e^{i k_{2} \theta} \varphi_{2}}\right]$. Obviously, by Lemma 3.4 it follows from (4.2) that

$$
\begin{aligned}
{\left[T_{e^{i k_{1} \theta} \varphi_{1}}, T_{e^{i k_{2} \theta} \varphi_{2}}\right] } & =\sum_{j=1}^{N} C_{k_{(j)}}\left(r^{\left|k_{(j)}\right|} e^{i k_{(j)} \theta}\right) \otimes\left(r^{\left|k_{(j)}-k_{1}-k_{2}\right|} e^{i\left(k_{(j)}-k_{1}-k_{2}\right) \theta}\right) \\
& +C_{-k_{(j)}+k_{1}+k_{2}}\left(r^{\left|-k_{(j)}+k_{1}+k_{2}\right|} e^{i\left(-k_{(j)}+k_{1}+k_{2}\right) \theta}\right) \otimes\left(r^{\left|-k_{(j)}\right|} e^{-i k_{(j)} \theta}\right)
\end{aligned}
$$

for nonzero constant $C_{k_{(j)}}=\left(\left|k_{(j)}-k_{1}-k_{2}\right|+1\right) C_{k_{(j)}}^{\prime}$, where $C_{k_{(j)}}^{\prime}$ is the coefficient satisfying

$$
\left[T_{e^{i k_{1} \theta} \varphi_{1}}, T_{e^{i k_{2} \theta} \varphi_{2}}\right]\left(r^{\left|k_{(j)}-k_{1}-k_{2}\right|} e^{i\left(k_{(j)}-k_{1}-k_{2}\right) \theta}\right)=\left.C_{k_{(j)}}^{\prime}\right|^{\left|k_{(j)}\right|} e^{i k_{(j)} \theta},
$$

and nonzero constant $C_{-k_{(j)}+k_{1}+k_{2}}=\left(\left|-k_{(j)}\right|+1\right) C_{-k_{(j)}+k_{1}+k_{2}}^{\prime}$, where $C_{-k_{(j)}+k_{1}+k_{2}}^{\prime}$ satisfies

$$
\left[T_{e^{i k_{1} \theta} \varphi_{1}}, T_{e^{i k_{2} \theta} \varphi_{2}}\right]\left(r^{\left|-k_{(j)}\right|} e^{-i k_{(j)} \theta}\right)=C_{-k_{(j)}+k_{1}+k_{2}}^{\prime} r^{\left|-k_{(j)}+k_{1}+k_{2}\right|} e^{i\left(-k_{(j)}+k_{1}+k_{2}\right) \theta} .
$$

According to Lemma 3.2, it follows $C_{k_{(j)}}^{\prime}=\lambda_{k_{2}, k_{(j)}-k_{1}-k_{2}} \lambda_{k_{1}, k_{(j)}-k_{1}}-\lambda_{k_{1}, k_{(j)}-k_{1}-k_{2}} \lambda_{k_{2}, k_{(j)}-k_{2}}$ and $C_{-k_{(j)}+k_{1}+k_{2}}^{\prime}=\lambda_{k_{2},-k_{(j)}} \lambda_{k_{1},-k_{(j)}+k_{2}}-\lambda_{k_{1},-k_{(j)}} \lambda_{k_{2},-k_{(j)}+k_{1}}$. Then by (3.1), it can easily be checked that

$$
\left(\left|k_{(j)}-k_{1}-k_{2}\right|+1\right) \lambda_{k_{2}, k_{(j)}-k_{1}-k_{2}} \lambda_{k_{1}, k_{(j)}-k_{1}}=\left(\left|-k_{(j)}\right|+1\right) \lambda_{k_{1},-k_{(j)}} \lambda_{k_{2},-k_{(j)}+k_{1}}
$$

and

$$
\left(\left|k_{(j)}-k_{1}-k_{2}\right|+1\right) \lambda_{k_{1}, k_{(j)}-k_{1}-k_{2}} \lambda_{k_{2}, k_{(j)}-k_{2}}=\left(\left|-k_{(j)}\right|+1\right) \lambda_{k_{2},-k_{(j)}} \lambda_{k_{1},-k_{(j)}+k_{2}},
$$

which implies

$$
\left(\left|k_{(j)}-k_{1}-k_{2}\right|+1\right) C_{k_{(j)}}^{\prime}=-\left(\left|-k_{(j)}\right|+1\right) C_{-k_{(j)}+k_{1}+k_{2}}^{\prime},
$$

and consequently, $C_{k_{(j)}}=-C_{-k_{(j)}+k_{1}+k_{2}}$. Then it follows from (4.6) that (4.3) holds.

Finally, observe that

$$
2 N_{1}+\left(k_{1}+k_{2}\right)=\left|k_{1}\right|+\left|k_{2}\right|,
$$

so (4.5) implies that $\operatorname{rank}\left(\left[T_{e^{i k_{1} \theta} \varphi_{1}}, T_{e^{i k_{2} \theta} \varphi_{2}}\right]\right) \leq\left|k_{1}\right|+\left|k_{2}\right|-1$ when $k_{1}+k_{2}$ is odd, and $\operatorname{rank}\left(\left[T_{e^{i k_{1} \theta} \varphi_{1}}, T_{e^{i k_{2} \theta} \varphi_{2}}\right]\right) \leq \max \left\{0,\left|k_{1}\right|+\left|k_{2}\right|-2\right\}$ when $k_{1}+k_{2}$ is even, as desired.

Remark 4.2. (1) It is obvious that the finite rank commutator $\left[T_{e^{i k_{1} \theta} \varphi_{1}}, T_{e^{i k_{2} \theta} \varphi_{2}}\right]$ can't have an odd rank, which corresponds to the result of [9]. Moreover, (4.3) implies that the canonical form of finite rank commutator of quasihomogeneous Toeplitz operators must exist always in pairs.

(2) One can also obtain further result by applying the adjoint operator. Notice that

$$
\left[T_{e^{i k_{1} \theta} \varphi_{1}}, T_{e^{i k_{2} \theta} \varphi_{2}}\right]^{*}=-\left[T_{e^{-i k_{1} \theta} \overline{\varphi_{1}}}, T_{e^{-i k_{2} \theta} \bar{\varphi}_{2}}\right],
$$

then it follows from (4.3) and Proposition 2.1 that

$$
\begin{aligned}
{\left[T_{e^{-i k_{1} \theta} \overline{\varphi_{1}}}, T_{e^{-i k_{2} \theta} \bar{\varphi}_{2}}\right]=\sum_{j=1}^{N} \overline{C_{k_{(j)}}}[} & \left(r^{\left|-k_{(j)}\right|} e^{-i k_{(j)} \theta}\right) \otimes\left(r^{\left|-k_{(j)}+k_{1}+k_{2}\right|} e^{i\left(-k_{(j)}+k_{1}+k_{2}\right) \theta}\right) \\
& \left.-\left(r^{\left|k_{(j)}-k_{1}-k_{2}\right|} e^{i\left(k_{(j)}-k_{1}-k_{2}\right) \theta}\right) \otimes\left(r^{\left|k_{(j)}\right|} e^{i k_{(j)} \theta}\right)\right] .
\end{aligned}
$$

The following corollary gives a complete description of the finite rank commutators of quasihomogeneous Toeplitz operators with some special degrees.

Corollary 4.3. Let $k \in \mathbb{Z}$, and let $\varphi_{1}, \varphi_{2}$ be two nonzero radial T-functions. Then on $L_{h}^{2}$, the following statements hold.

(a) The commutator $\left[T_{\varphi_{1}}, T_{e^{i k \theta} \varphi_{2}}\right]$ has finite rank if and only if $k=0$ or $\varphi_{1}$ is a constant.

(b) If $k \neq 0$, then the commutator $\left[T_{e^{i k \theta} \varphi_{1}}, T_{e^{i k \theta} \varphi_{2}}\right]$ has finite rank if and only if $\varphi_{1}=C \varphi_{2}$ for some constant $C$. 
(c) If $k \neq 0$, then the commutator $\left[T_{e^{i k \theta} \varphi_{1}}, T_{e^{-i k \theta} \varphi_{2}}\right]$ has finite rank if and only if $|k|=1$ and $\varphi_{1} *_{M} \varphi_{2}=C\left(r *_{M} r^{-1}\right)$ for some constant $C$.

In each condition, the rank of the commutator is zero.

Proof. First we suppose $\left[T_{\varphi_{1}}, T_{e^{i k \theta} \varphi_{2}}\right]$ has finite rank, then Proposition 4.1 shows that

$$
2(n+k+1) \widehat{\varphi_{1}}(2 n+2 k+2) \widehat{\varphi_{2}}(2 n+k+2)=2(n+1) \widehat{\varphi_{1}}(2 n+2) \widehat{\varphi_{2}}(2 n+k+2)
$$

holds for any natural number $n \geq \max \{0,-k\}$. Using the same reasoning as in the proof of [26. Proposition 6], one can easily get that either $k=0$ or $\varphi_{1}$ is a constant.

Next, suppose $\left[T_{e^{i k \theta} \varphi_{1}}, T_{e^{i k \theta} \varphi_{2}}\right]$ has finite rank. Without loss of generality, we can assume that $k>0$, for otherwise we could take the adjoints. Then Proposition 4.1 shows that

$$
\widehat{\varphi_{1}}(2 n+3 k+2) \widehat{\varphi_{2}}(2 n+k+2)=\widehat{\varphi_{1}}(2 n+k+2) \widehat{\varphi_{2}}(2 n+3 k+2)
$$

for any $n \in \mathbb{N}$, which is the same as Equation (3.4) of [20, and hence $\varphi_{1}=C \varphi_{2}$.

Finally, suppose $\left[T_{e^{i k \theta} \varphi_{1}}, T_{e^{-i k \theta} \varphi_{2}}\right]$ has finite rank. Similarly, we assume $k>0$. Then Proposition 4.1 shows that

$$
\begin{aligned}
& (2 n-2 k+2)(2 n+2) \widehat{\varphi_{1}}(2 n-k+2) \widehat{\varphi_{2}}(2 n-k+2) \\
& =(2 n+2)(2 n+2 k+2) \widehat{\varphi_{1}}(2 n+k+2) \widehat{\varphi_{2}}(2 n+k+2)
\end{aligned}
$$

for any natural number $n \geq k$. We now proceed as in the proof of condition (b) of 20, Proposition 3.4], then $(z-k)(z+k) \widehat{\varphi_{1}}(z) \widehat{\varphi_{2}}(z)=C$ for some nonzero constant $C$. On the other hand, since $\varphi_{1}, \varphi_{2} \in L^{1}([0,1], r d r)$, we get $\varphi_{1} *_{M} \varphi_{2} \in L^{1}([0,1], r d r)$. Therefore

$$
\widehat{\varphi_{1} *_{M} \varphi_{2}}(z)=\widehat{\varphi_{1}}(z) \widehat{\varphi_{2}}(z)=\frac{C}{(z-k)(z+k)}
$$

must be well defined on $\{z: \operatorname{Re} z \geq 2\}$, which implies $k=1$ and $\varphi_{1} *_{M} \varphi_{2}=C\left(r *_{M} r^{-1}\right)$.

The sufficiency of conditions (a)-(c) is an immediate consequence of [20, Proposition 3.4]. Furthermore, the commutator is zero in each condition. This completes the proof.

Now we are ready to state and prove our main theorem in this section, which completely solves the finite rank problem of the commutator $\left[T_{e^{i k_{1} \theta} r^{m}}, T_{e^{i k_{2} \theta} \varphi}\right]$ on $L_{h}^{2}$.

Theorem 4.4. Let $k_{1}, k_{2} \in \mathbb{Z}$, and let $m \in \mathbb{R}, m \geq-1$. Then for a nonzero radial $T$-function $\varphi(r)$ on $D$, the commutator $\left[T_{e^{i k_{1} \theta} r^{m}}, T_{e^{i k_{2} \theta} \varphi}\right]$ has finite rank on $L_{h}^{2}$ if and only if one of the following conditions holds:

(1) $k_{1}=m=0$.

(2) $k_{2}=0$ and $\varphi=C$.

(3) $k_{1}=k_{2}=0$.

(4) $k_{1}=k_{2} \neq 0$ and $\varphi=C r^{m}$.

(5) $k_{1} k_{2}=-1$ and $\varphi=C\left(\frac{m+1}{2} r^{-1}-\frac{m-1}{2} r\right)$.

(6) $k_{1} k_{2}<-1,\left|k_{2}\right| \geq 2, m+\left|k_{1}\right|=0$ and $\varphi(r)=C r^{\left|k_{2}\right|}$.

(7) $k_{1} k_{2}<-1,\left|k_{2}\right|=1$ and $\widehat{\varphi}(z)=C \frac{\Gamma\left(\frac{z-1}{2\left|k_{1}\right|}\right) \Gamma\left(\frac{z+m+\left|k_{1}\right|+1}{2\left|k_{1}\right|}\right)}{\Gamma\left(\frac{z+2\left|k_{1}\right|+1}{2\left|k_{1}\right|}\right) \Gamma\left(\frac{z+m+k_{1} \mid-1}{2\left|k_{1}\right|}\right)}$.

(8) $k_{1} k_{2}>0, k_{1} \neq k_{2},\left|k_{2}\right|<m+\left|k_{1}\right|+2$ and $\widehat{\varphi}(z)=C \frac{\Gamma\left(\frac{z+\left|k_{2}\right|}{2 k_{1} \mid}\right) \Gamma\left(\frac{z+m+\left|k_{1}\right|-\left|k_{2}\right|}{2\left|k_{1}\right|}\right)}{\Gamma\left(\frac{z+2\left|k_{1}\right|-\left|k_{2}\right|}{2\left|k_{1}\right|}\right) \Gamma\left(\frac{z+m+\left|k_{1}\right|+\left|k_{2}\right|}{2\left|k_{1}\right|}\right)}$.

(9) $k_{1} k_{2}>0,\left|k_{2}\right| \geq m+\left|k_{1}\right|+2, m=(2 n+1)\left|k_{1}\right|$ for some $n \in \mathbb{N}$ and

$$
\widehat{\varphi}(z)=C \frac{\left(\frac{z-\left|k_{2}\right|}{2\left|k_{1}\right|}+1\right)\left(\frac{z-\left|k_{2}\right|}{2\left|k_{1}\right|}+2\right) \cdots\left(\frac{z-\left|k_{2}\right|}{2\left|k_{1}\right|}+n\right)}{\frac{z+\left|k_{2}\right|}{2\left|k_{1}\right|}\left(\frac{z+\left|k_{2}\right|}{2\left|k_{1}\right|}+1\right) \cdots\left(\frac{z+\left|k_{2}\right|}{2\left|k_{1}\right|}+n\right)} .
$$

In each condition (1)-(5), $\operatorname{rank}\left(\left[T_{e^{i k_{1} \theta} r^{m}}, T_{e^{i k_{2} \theta} \varphi}\right]\right)=0$. In each condition (6)-(9),

$$
\operatorname{Ran}\left(\left[T_{e^{i k_{1} \theta} r^{m}}, T_{e^{i k_{2} \theta} \varphi}\right]\right)=\operatorname{span}\left\{r^{|k|} e^{i k \theta}: k \in \Lambda_{1}\right\}
$$


and

$$
\begin{aligned}
{\left[T_{e^{i k_{1} \theta} r^{m}}, T_{e^{i k_{2} \theta} \varphi}\right]=\sum_{k \in \Lambda_{\frac{1}{2}}} C_{k}[} & \left(r^{|k|} e^{i k \theta}\right) \otimes\left(r^{\left|k-k_{1}-k_{2}\right|} e^{i\left(k-k_{1}-k_{2}\right) \theta}\right) \\
& \left.-\left(r^{\left|-k+k_{1}+k_{2}\right|} e^{i\left(-k+k_{1}+k_{2}\right) \theta}\right) \otimes\left(r^{|-k|} e^{-i k \theta}\right)\right]
\end{aligned}
$$

for some nonzero constant $C_{k}$. Furthermore, $\operatorname{rank}\left(\left[T_{e^{i k_{1} \theta} r^{m}}, T_{e^{i k_{2} \theta} \varphi}\right]\right)=\left|k_{1}\right|+\left|k_{2}\right|-1$ when $k_{1}+k_{2}$ is odd, and $\operatorname{rank}\left(\left[T_{e^{i k_{1} \theta} r^{m}}, T_{e^{i k_{2} \theta} \varphi}\right]\right)=\left|k_{1}\right|+\left|k_{2}\right|-2$ when $k_{1}+k_{2}$ is even.

Proof. If $k_{1} k_{2}=0$ or $\left|k_{1}\right|=\left|k_{2}\right|$, then Corollary 4.3 shows that the commutator $\left[T_{e^{i k_{1} \theta} r^{m}}, T_{e^{i k_{2} \theta} \varphi}\right]$ has finite rank if and only if one of the conditions (1)-(5) holds, and $\operatorname{rank}\left(\left[T_{e^{i k_{1} \theta} r^{m}}, T_{e^{i k_{2} \theta} \varphi}\right]\right)=$ 0 in each condition.

Now, we suppose $k_{1} k_{2} \neq 0$ and $\left|k_{1}\right| \neq\left|k_{2}\right|$. Without loss of generality, we can also assume that $k_{1}>0$, for otherwise we could take the adjoints. Then by Proposition 4.1, the commutator $\left[T_{e^{i k_{1} \theta} r^{m}}, T_{e^{i k_{2} \theta} \varphi}\right]$ has finite rank if and only if

$$
\begin{aligned}
& 2\left(n+k_{2}+1\right) \widehat{r^{m}}\left(2 n+k_{1}+2 k_{2}+2\right) \widehat{\varphi}\left(2 n+k_{2}+2\right) \\
& =2\left(n+k_{1}+1\right) \widehat{r^{m}}\left(2 n+k_{1}+2\right) \widehat{\varphi}\left(2 n+2 k_{1}+k_{2}+2\right)
\end{aligned}
$$

holds for any natural number $n \geq N_{1}=\max \left\{0,-k_{2}\right\}$. Then it follows that

$$
\widehat{\varphi}\left(2 n+2 k_{1}+k_{2}+2\right)=\widehat{\varphi}\left(2 n+k_{2}+2\right) \frac{\left(2 n+2 k_{2}+2\right)\left(2 n+k_{1}+m+2\right)}{\left(2 n+2 k_{1}+2\right)\left(2 n+k_{1}+2 k_{2}+m+2\right)},
$$

which is the same as Equation (2.4) of [16, so one can get

$$
\widehat{\varphi}(z)=C \frac{\Gamma\left(\frac{z+k_{2}}{2 k_{1}}\right) \Gamma\left(\frac{z+m+k_{1}-k_{2}}{2 k_{1}}\right)}{\Gamma\left(\frac{z+2 k_{1}-k_{2}}{2 k_{1}}\right) \Gamma\left(\frac{z+m+k_{1}+k_{2}}{2 k_{1}}\right)}
$$

for some nonzero constant $C$. Thus, according to Lemma 3.7 the commutator $\left[T_{e^{i k_{1} \theta} r^{m}}, T_{e^{i k_{2} \theta} \varphi}\right]$ has finite rank if and only if one of the conditions (6)-(9) holds.

Comparing with Proposition 4.1 this theorem will be proved if we can show that (4.7) holds in each condition (6)-(9). Moreover, according to the proof of Proposition 4.1, we only need to show that $r^{|k|} e^{i k \theta} \in \operatorname{Ran}\left(\left[T_{e^{i k_{1} \theta} r^{m}}, T_{e^{i k_{2} \theta} \varphi_{2}}\right]\right)$ holds for any $k \in \Lambda_{\frac{1}{2}}$, or equivalently,

$$
\left[T_{e^{i k_{1} \theta} r^{m}}, T_{e^{i k_{2} \theta} \varphi}\right]\left(r^{\left|k-k_{1}-k_{2}\right|} e^{i\left(k-k_{1}-k_{2}\right) \theta}\right)=C_{k} r^{|k|} e^{i k \theta}
$$

holds for some nonzero constant $C_{k}$. Recall that $k \in \Lambda_{\frac{1}{2}}$ implies $\frac{-k_{1}-k_{2}}{2}<k-k_{1}-k_{2} \leq N_{1}-1$. So it suffices to prove that, for any $l \in\left\{\left[\frac{-k_{1}-k_{2}}{2}\right], \cdots, N_{1}-1\right\}$, where $[\cdot]$ denotes the greatest integer function,

$$
\left[T_{e^{i k_{1} \theta} r^{m}}, T_{e^{i k_{2} \theta} \varphi}\right]\left(r^{|l|} e^{i l \theta}\right)=0 \Longleftrightarrow l=\frac{-k_{1}-k_{2}}{2} .
$$

To show this we need to discuss four cases.

Case 1. Suppose $k_{1}=-m=1, k_{2} \leq-2$. Thus $N_{1}=-k_{2}$. So for any $\left[\frac{-1-k_{2}}{2}\right] \leq n \leq-1-k_{2}$, by Lemma 3.1 we get

$$
\begin{aligned}
{\left[T_{e^{i \theta} r^{-1}}, T_{e^{i k_{2} \theta} \varphi}\right]\left(z^{n}\right)=0 \Longleftrightarrow } & \left(-k_{2}-n+1\right) \widehat{r^{m}}\left(-k_{1}-2 k_{2}-2 n+2\right) \widehat{\varphi}\left(-k_{2}+2\right) \\
& =\left(k_{1}+n+1\right) \widehat{r^{m}}\left(2 n+k_{1}+2\right) \widehat{\varphi}\left(-k_{2}+2\right) \\
& \Longleftrightarrow \frac{-k_{2}-n+1}{-2 k_{2}-2 n}=\frac{n+2}{2 n+2} \Longleftrightarrow n=\frac{-1-k_{2}}{2},
\end{aligned}
$$

as desired. 
Case 2. Suppose $k_{1}>1$ and $k_{2}=-1$. Thus $N_{1}=1$. So for any $0 \leq n \leq\left[\frac{k_{1}+k_{2}}{2}\right]$, by Lemma 3.1 we get

$$
\begin{aligned}
{\left[T_{e^{i k_{1} \theta} r^{m}}, T_{e^{i k_{2} \theta} \varphi}\right]\left(\bar{z}^{n}\right)=0 \Longleftrightarrow } & \left(n-k_{2}+1\right) \widehat{r^{m}}\left(k_{1}+2\right) \widehat{\varphi}\left(2 n-k_{2}+2\right) \\
& =\left(k_{1}-n+1\right) \widehat{\varphi}\left(2 k_{1}+k_{2}-2 n+2\right) \widehat{r^{m}}\left(k_{1}+2\right) \\
\Longleftrightarrow & \Longleftrightarrow F(n)=F\left(k_{1}+k_{2}-n\right),
\end{aligned}
$$

where

$$
F(x)=2\left(x-k_{2}+1\right) \widehat{\varphi}\left(2 x-k_{2}+2\right) .
$$

Let $a=\frac{k_{2}}{k_{1}}$ and $b=\frac{m+k_{1}}{2 k_{1}}$. Then by (4.9), we get

$$
\begin{aligned}
F(x) & =2 k_{1} C \frac{2 x-2 k_{2}+2}{2 k_{1}} \frac{\Gamma\left(\frac{2 x+2}{2 k_{1}}\right) \Gamma\left(\frac{2 x+m+k_{1}-2 k_{2}+2}{2 k_{1}}\right)}{\Gamma\left(\frac{2 x+2 k_{1}-2 k_{2}+2}{2 k_{1}}\right) \Gamma\left(\frac{2 x+m+k_{1}+2}{2 k_{1}}\right)} \\
& =2 k_{1} C \frac{\Gamma\left(\frac{x+1}{k_{1}}\right) \Gamma\left(\frac{x+1}{k_{1}}+b-a\right)}{\Gamma\left(\frac{x+1}{k_{1}}-a\right) \Gamma\left(\frac{x+1}{k_{1}}+b\right)} .
\end{aligned}
$$

Note that $a<0$ and $b>0$, so condition (b) of Lemma 3.5 implies that $F(x)$ is strictly monotone on $(-1,+\infty)$. Thus (4.10) holds if and only if $n=\frac{k_{1}+k_{2}}{2}$, as desired.

Case 3. Suppose $k_{1}>0, k_{2}>0$ and $k_{1}<k_{2}$. Thus $N_{1}=0$. Observe that both condition (8) and condition (9) imply that $m+k_{1} \neq 0$. So for any $k_{1} \leq n \leq\left[\frac{k_{1}+k_{2}}{2}\right]$, by Lemma 3.1 we get

$$
\begin{aligned}
{\left[T_{e^{i k_{1} \theta} r^{m}}, T_{e^{i k_{2} \theta} \varphi}\right]\left(\bar{z}^{n}\right)=0 } & \Longleftrightarrow\left(k_{2}-n+1\right) \widehat{r^{m}}\left(k_{1}+2 k_{2}-2 n+2\right) \widehat{\varphi}\left(k_{2}+2\right) \\
& =\left(n-k_{1}+1\right) \widehat{r^{m}}\left(2 n-k_{1}+2\right) \widehat{\varphi}\left(k_{2}+2\right) \\
& \Longleftrightarrow \frac{k_{2}-n+1}{m+k_{1}+2 k_{2}-2 n+2}=\frac{n-k_{1}+1}{m+2 n-k_{1}+2} \\
& \Longleftrightarrow \frac{m+k_{1}}{k_{2}-n+1}=\frac{m+k_{1}}{n-k_{1}+1} \Longleftrightarrow n=\frac{k_{1}+k_{2}}{2} .
\end{aligned}
$$

Next, for any $1 \leq n<k_{1}$, by Lemma 3.1 we get

$$
\begin{aligned}
{\left[T_{e^{i k_{1} \theta} r^{m}}, T_{e^{i k_{2} \theta} \varphi}\right]\left(\bar{z}^{n}\right)=0 \Longleftrightarrow } & 2\left(k_{2}-n+1\right) \widehat{r^{m}}\left(k_{1}+2 k_{2}-2 n+2\right) \widehat{\varphi}\left(k_{2}+2\right) \\
& =2\left(k_{1}-n+1\right) \widehat{r^{m}}\left(k_{1}+2\right) \widehat{\varphi}\left(2 k_{1}+k_{2}-2 n+2\right) \\
\Longleftrightarrow & \frac{2\left(k_{2}-n+1\right)}{m+k_{1}+2 k_{2}-2 n+2} \frac{\Gamma\left(\frac{2 k_{2}+2}{2 k_{1}}\right) \Gamma\left(\frac{m+k_{1}+2}{2 k_{1}}\right)}{\Gamma\left(\frac{2 k_{1}+2}{2 k_{1}}\right) \Gamma\left(\frac{m+k_{1}+2 k_{2}+2}{2 k_{1}}\right)} \\
& =\frac{2\left(k_{1}-n+1\right)}{m+k_{1}+2} \frac{\Gamma\left(\frac{2 k_{1}+2 k_{2}-2 n+2}{2 k_{1}}\right) \Gamma\left(\frac{m+3 k_{1}-2 n+2}{2 k_{1}}\right)}{\Gamma\left(\frac{4 k_{1}-2 n+2}{2 k_{1}}\right) \Gamma\left(\frac{m+3 k_{1}+2 k_{2}-2 n+2}{2 k_{1}}\right)} .
\end{aligned}
$$

Let $a=\frac{k_{2}}{k_{1}}, b=\frac{m+k_{1}}{2 k_{1}}$ and $x=\frac{n}{k_{1}}$. Then the above equation becomes

$$
\begin{aligned}
& \frac{\frac{1}{k_{1}}-x+a}{\frac{1}{k_{1}}-x+b+a} \frac{\Gamma\left(\frac{1}{k_{1}}+a\right) \Gamma\left(\frac{1}{k_{1}}+b\right)}{\Gamma\left(\frac{1}{k_{1}}+1\right) \Gamma\left(\frac{1}{k_{1}}+b+a\right)}=\frac{\frac{1}{k_{1}}-x+1}{\frac{1}{k_{1}}+b} \frac{\Gamma\left(\frac{1}{k_{1}}-x+a+1\right) \Gamma\left(\frac{1}{k_{1}}-x+b+1\right)}{\Gamma\left(\frac{1}{k_{1}}-x+2\right) \Gamma\left(\frac{1}{k_{1}}-x+b+a+1\right)} \\
& \Longleftrightarrow \frac{\Gamma\left(\frac{1}{k_{1}}-x+a\right) \Gamma\left(\frac{1}{k_{1}}-x+b+1\right)}{\Gamma\left(\frac{1}{k_{1}}-x+1\right) \Gamma\left(\frac{1}{k_{1}}-x+b+a\right)}=\frac{\Gamma\left(\frac{1}{k_{1}}+a\right) \Gamma\left(\frac{1}{k_{1}}+b+1\right)}{\Gamma\left(\frac{1}{k_{1}}+1\right) \Gamma\left(\frac{1}{k_{1}}+b+a\right)} \\
& \Longleftrightarrow G\left(x-\frac{1}{k_{1}}\right)=G\left(-\frac{1}{k_{1}}\right),
\end{aligned}
$$


where the function $G(x)$ is defined by (3.6). Obviously, this is contradict to condition (b) of Lemma 3.6 since $a>1, b>0$ and $x \in(0,1)$, as desired.

Case 4. Suppose $k_{1}>0, k_{2}>0$ and $k_{1}>k_{2}$. Thus $N_{1}=0$. So for any $k_{2} \leq n \leq\left[\frac{k_{1}+k_{2}}{2}\right]$, by Lemma 3.1 we get

$$
\begin{aligned}
{\left[T_{e^{i k_{1} \theta} r^{m}}, T_{e^{i k_{2} \theta} \varphi}\right]\left(\bar{z}^{n}\right)=0 \Longleftrightarrow } & 2\left(n-k_{2}+1\right) \widehat{r^{m}}\left(k_{1}+2\right) \widehat{\varphi}\left(2 n-k_{2}+2\right) \\
& =2\left(k_{1}-n+1\right) \widehat{\varphi}\left(2 k_{1}+k_{2}-2 n+2\right) \widehat{r^{m}}\left(k_{1}+2\right) \\
& \Longleftrightarrow F(n)=F\left(k_{1}+k_{2}-n\right),
\end{aligned}
$$

where $F(x)$ is also defined by (4.11). Here $a=\frac{k_{2}}{k_{1}}>0$ and $b=\frac{m+k_{1}}{2 k_{1}}>0$. Then condition (a) of Lemma 3.5 implies that $F(x)$ is strictly monotone on $\left(k_{2}-1,+\infty\right)$, and hence (4.13) holds if and only if $n=\frac{k_{1}+k_{2}}{2}$, as desired.

Next, for any $1 \leq n<k_{2}$, by Lemma 3.1 we get

$$
\begin{aligned}
{\left[T_{e^{i k_{1} \theta} r^{m}}, T_{e^{i k_{2} \theta} \varphi}\right]\left(\bar{z}^{n}\right)=0 \Longleftrightarrow } & 2\left(k_{2}-n+1\right) \widehat{r^{m}}\left(k_{1}+2 k_{2}-2 n+2\right) \widehat{\varphi}\left(k_{2}+2\right) \\
& =2\left(k_{1}-n+1\right) \widehat{r^{m}}\left(k_{1}+2\right) \widehat{\varphi}\left(2 k_{1}+k_{2}-2 n+2\right),
\end{aligned}
$$

which is the same as (4.12). Using the same notation, we get that the above equation is also equivalent to $G\left(x-\frac{1}{k_{1}}\right)=G\left(-\frac{1}{k_{1}}\right)$. Here $a=\frac{k_{2}}{k_{1}} \in(0,1), b=\frac{m+k_{1}}{2 k_{1}}>0$ and $x=\frac{n}{k_{1}} \in(0, a)$. Obviously, this is contradict to condition (a) of Lemma 3.6 This completes the proof.

Below we present the examples of nonzero commutators of finite rank, corresponding to the conditions (6)-(9) of Theorem 4.4 .

Example 4.5. Let $\varphi(r)$ be a nonzero radial T-function on $D$, then on $L_{h}^{2}$, the following statements hold.

(1) The commutator $\left[T_{e^{i \theta} r^{-1}}, T_{e^{-3 i \theta} \varphi(r)}\right]$ has finite rank if and only if $\varphi(r)=C r^{3}$. In this case, $\left[T_{e^{i \theta} r^{-1}}, T_{e^{-3 i \theta} \varphi(r)}\right]=\frac{1}{2} C\left(1 \otimes z^{2}-\bar{z}^{2} \otimes 1\right)$.

(2) The commutator $\left[T_{e^{2 i \theta} r^{6}}, T_{e^{-i \theta} \varphi(r)}\right]$ has finite rank if and only if $\varphi(r)=C\left(\frac{3}{r}-r^{3}\right)$. In this case, $\left[T_{e^{2 i \theta} r^{6}}, T_{e^{-i \theta} \varphi(r)}\right]=-\frac{19}{30} C(1 \otimes \bar{z}-z \otimes 1)$.

(3) Let $m \in \mathbb{R}, m \geq-1$. Then the commutator $\left[T_{e^{i \theta} r^{m}}, T_{e^{2 i \theta} \varphi(r)}\right]$ has finite rank if and only if $m>-1$ and $\varphi(r)=C\left[(m+1) r^{m+1}-(m-1) r^{m-1}\right]$.

In this case, $\left[T_{e^{i \theta} r^{m}}, T_{e^{2 i \theta} \varphi(r)}\right]=-\frac{192(m+1)}{(m+5)^{2}(m+3)^{2}} C\left(z \otimes \bar{z}^{2}-z^{2} \otimes \bar{z}\right)$.

(4) The commutator $\left[T_{e^{i \theta} r^{3}}, T_{e^{6 i \theta} \varphi(r)}\right]$ has finite rank if and only if $\varphi(r)=C\left[6 r^{8}-5 r^{6}\right]$. In this case, $\left[T_{e^{i \theta} r^{3}}, T_{e^{6 i \theta} \varphi(r)}\right]=C\left[-\frac{5}{24}\left(z \otimes \bar{z}^{6}-z^{6} \otimes \bar{z}\right)-\frac{27}{196}\left(z^{2} \otimes \bar{z}^{5}-z^{5} \otimes \bar{z}^{2}\right)\right.$ $\left.-\frac{1}{21}\left(z^{3} \otimes \bar{z}^{4}-z^{4} \otimes \bar{z}^{3}\right)\right]$.

Next, we show some interesting applications of Theorem 4.4.

Corollary 4.6. Let $k_{1}, k_{2} \in \mathbb{Z}$ such that $k_{1}>0$ or $k_{1}=-1$, and let $\varphi(r)$ be a nonzero radial $T$-function on $D$. Then on $L_{h}^{2}$, the following statements hold.

(a) The commutator $\left[T_{e^{i k_{1} \theta} r^{k_{1}}}, T_{e^{i k_{2} \theta} \varphi}\right]$ has finite rank if and only if

$$
k_{2}>-2 \text { and } \varphi(r)=C r^{k_{2}} .
$$

(b) The commutator $\left[T_{e^{-i k_{1} \theta} r^{k_{1}}}, T_{e^{i k_{2} \theta} \varphi}\right]$ has finite rank if and only if

$$
k_{2}<2 \text { and } \varphi(r)=\mathrm{Cr}^{-k_{2}} .
$$

Proof. If $k_{1}>0$, then by Theorem 4.4, the commutator $\left[T_{e^{i k_{1} \theta} r^{k_{1}}}, T_{e^{i k_{2} \theta} \varphi}\right]$ has finite rank if and only if $k_{2}$ satisfies one of the conditions (2), (4)-(5) and (7)-(9), and $\widehat{\varphi}(z)=\frac{C}{z+k_{2}}$. In other words, $k_{2}>-2$ and $\varphi(r)=C r^{k_{2}}$.

Similarly, if $k_{1}=-1$, then $\left[T_{e^{i k_{1} \theta} r^{k_{1}}}, T_{e^{i k_{2} \theta} \varphi}\right]$ has finite rank if and only if one of the conditions (2) and (4)-(6) holds, which also implies that $k_{2}>-2$ and $\varphi(r)=C r^{k_{2}}$.

Combining condition (a) with the use of adjoint operators, one can get condition (b) holds. 
Corollary 4.7. Let $k_{1}, k_{2} \in \mathbb{Z}$ such that $k_{1} k_{2} \neq 0$, and let $m_{1}, m_{2} \in \mathbb{R}$ such that greater than or equal to -1 . Then the commutator $\left[T_{e^{i k_{1} \theta} r^{m_{1}}}, T_{e^{i k_{2} \theta} r^{m_{2}}}\right]$ has finite rank on $L_{h}^{2}$ if and only if one of the following conditions holds:

(1) $k_{1}=k_{2}$ and $m_{1}=m_{2}$.

(2) $k_{1}=m_{1}$ and $k_{2}=m_{2}$.

(3) $k_{1}=-m_{1}$ and $k_{2}=-m_{2}$.

Proof. Without loss of generality, we can assume that $k_{1}>0$. Since $k_{2} \neq 0$ and

$$
\frac{k_{2}}{2 k_{1}}+\frac{m_{1}+k_{1}-k_{2}}{2 k_{1}}=\frac{2 k_{1}-k_{2}}{2 k_{1}}+\frac{m_{1}+k_{1}+k_{2}}{2 k_{1}}-1,
$$

one can easily see that

$$
\widehat{\varphi}(z)=C \frac{\Gamma\left(\frac{z+k_{2}}{2 k_{1}}\right) \Gamma\left(\frac{z+m_{1}+k_{1}-k_{2}}{2 k_{1}}\right)}{\Gamma\left(\frac{z+2 k_{1}-k_{2}}{2 k_{1}}\right) \Gamma\left(\frac{z+m_{1}+k_{1}+k_{2}}{2 k_{1}}\right)}
$$

is a proper rational function with the degree of the denominator one if and only if

$$
\frac{2 k_{1}-2 k_{2}}{2 k_{1}}=0, \quad \frac{k_{1}-m_{1}}{2 k_{1}}=0 \text { or } \frac{m_{1}+k_{1}}{2 k_{1}}=0 .
$$

Therefore, $\widehat{\varphi}(z)=\widehat{r^{m_{2}}}(z)=\frac{1}{z+m_{2}}$ holds if and only if one of the following conditions holds:

(1) $k_{1}=k_{2}>0$ and $m_{1}=m_{2}$.

(2) $k_{1}=m_{1}>0$ and $k_{2}=m_{2}$.

(3) $k_{1}=-m_{1}=1$ and $k_{2}=-m_{2}$.

Then by Theorem 4.4, the desired result is obvious.

Remark 4.8. By Theorem 4.4, one can easily get the range, the canonical form and the rank of the finite rank commutator appeared in Corollary 4.6 and Corollary 4.7. For example, for positive natural numbers $k_{1}$ and $k_{2}$, we have $\Lambda_{1}=\left\{k \in \mathbb{Z}: 1 \leq k \leq k_{1}+k_{2}-1, k \neq \frac{k_{1}+k_{2}}{2}\right\}$, and hence

$$
\left[T_{z^{k_{1}}}, T_{z^{k_{2}}}\right]=\sum_{k \in \Lambda_{1}} C_{k} z^{k_{1}+k_{2}-k} \otimes \bar{z}^{k},
$$

which is the same as [9. Proposition 2.3]. We would like to point out that in [9] the authors first got (4.14) by the direct calculation, taking on the reproducing kernel, then they showed that the commutator $\left[T_{z^{k_{1}}}, T_{z^{k_{2}}}\right]$ has finite rank. So the method used in this paper is quite different from that in 9 .

\section{RANKS OF Generalized SEMicommutators ON $L_{h}^{2}$}

In this section, we will discuss the finite rank generalized semicommutators of quasihomogeneous Toeplitz operators on $L_{h}^{2}$. Obviously, if $T_{e^{i k_{1} \theta} \varphi_{1}} T_{e^{i k_{2} \theta} \varphi_{2}}-T_{f}$ has finite rank, then $f$ must be quasihomogeneous of degree $k_{1}+k_{2}$. Therefore, it suffices to consider the generalized semicommutator of the form $T_{e^{i k_{1} \theta} \varphi_{1}} T_{e^{i k_{2} \theta} \varphi_{2}}-T_{e^{i\left(k_{1}+k_{2}\right) \theta} \psi}$ in this section. Throughout the rest part of this paper, we also define

$$
N_{2}=\max \left\{0,-k_{2},-k_{1}-k_{2}\right\} \text { and } N_{3}=\max \left\{0, k_{2}, k_{1}+k_{2}\right\} .
$$

Then we write

$$
\Lambda_{2}=\left\{k \in \mathbb{Z}:-N_{3}+k_{1}+k_{2}+1 \leq k \leq N_{2}+k_{1}+k_{2}-1\right\} .
$$

First we show the general result of the finite rank generalized semicommutators of two quasihomogeneous Toeplitz operators.

Proposition 5.1. Let $k_{1}, k_{2} \in \mathbb{Z}$, and let $\varphi_{1}, \varphi_{2}$ and $\psi$ be radial T-functions. Then the generalized semicommutator $T_{e^{i k_{1} \theta} \varphi_{1}} T_{e^{i k_{2} \theta} \varphi_{2}}-T_{e^{i\left(k_{1}+k_{2}\right) \theta} \psi}$ has finite rank on $L_{h}^{2}$ if and only if

$$
2\left(n+k_{2}+1\right) \widehat{\varphi_{1}}\left(2 n+k_{1}+2 k_{2}+2\right) \widehat{\varphi_{2}}\left(2 n+k_{2}+2\right)=\widehat{\psi}\left(2 n+k_{1}+k_{2}+2\right)
$$


holds for any natural number $n \geq N_{2}$, and

$$
2\left(n-k_{2}+1\right) \widehat{\varphi_{1}}\left(2 n-k_{1}-2 k_{2}+2\right) \widehat{\varphi_{2}}\left(2 n-k_{2}+2\right)=\widehat{\psi}\left(2 n-k_{1}-k_{2}+2\right)
$$

holds for any natural number $n \geq N_{3}$. In this case,

$$
\operatorname{Ran}\left(T_{e^{i k_{1} \theta} \varphi_{1}} T_{e^{i k_{2} \theta} \varphi_{2}}-T_{e^{i\left(k_{1}+k_{2}\right) \theta} \psi}\right)=\operatorname{span}\left\{r^{\left|k_{(1)}\right|} e^{i k_{(1)} \theta}, r^{\left|k_{(2)}\right|} e^{i k_{(2)} \theta}, \cdots, r^{\left|k_{(N)}\right|} e^{i k_{(N)} \theta}\right\}
$$

for some $k_{(1)}, \cdots, k_{(N)} \in \Lambda_{2}$, and

$$
T_{e^{i k_{1} \theta} \varphi_{1}} T_{e^{i k_{2} \theta} \varphi_{2}}-T_{e^{i\left(k_{1}+k_{2}\right) \theta} \psi}=\sum_{j=1}^{N} C_{k_{(j)}}\left(r^{\left|k_{(j)}\right|} e^{i k_{(j)} \theta}\right) \otimes\left(r^{\left|k_{(j)}-k_{1}-k_{2}\right|} e^{i\left(k_{(j)}-k_{1}-k_{2}\right) \theta}\right)
$$

for some nonzero constant $C_{k_{(j)}}$. Furthermore,

$$
\operatorname{rank}\left(T_{e^{i k_{1} \theta} \varphi_{1}} T_{e^{i k_{2} \theta} \varphi_{2}}-T_{e^{i\left(k_{1}+k_{2}\right) \theta} \psi}\right) \leq \max \left\{0,\left|k_{1}\right|-1,\left|k_{2}\right|-1,\left|k_{1}+k_{2}\right|-1\right\} .
$$

Proof. By Lemma 3.1 we get

$$
\begin{aligned}
& \left(T_{e^{i k_{1} \theta} \varphi_{1}} T_{e^{i k_{2} \theta} \varphi_{2}}-T_{e^{i\left(k_{1}+k_{2}\right)} \psi}\right)\left(z^{n}\right) \\
& =\left[2\left(n+k_{2}+1\right) \widehat{\varphi_{1}}\left(2 n+k_{1}+2 k_{2}+2\right) \widehat{\varphi_{2}}\left(2 n+k_{2}+2\right)-\widehat{\psi}\left(2 n+k_{1}+k_{2}+2\right)\right] \\
& \quad \times 2\left(n+k_{1}+k_{2}+1\right) z^{n+k_{1}+k_{2}}
\end{aligned}
$$

holds for any natural number $n \geq N_{2}$, and

$$
\begin{aligned}
& \left(T_{e^{i k_{1} \theta} \varphi_{1}} T_{e^{i k_{2} \theta} \varphi_{2}}-T_{e^{i\left(k_{1}+k_{2}\right)} \psi}\right)\left(\bar{z}^{n}\right) \\
& =\left[2\left(n-k_{2}+1\right) \widehat{\varphi_{1}}\left(2 n-k_{1}-2 k_{2}+2\right) \widehat{\varphi_{2}}\left(2 n-k_{2}+2\right)-\widehat{\psi}\left(2 n-k_{1}-k_{2}+2\right)\right] \\
& \quad \times 2\left(n-k_{1}-k_{2}+1\right) \bar{z}^{n-k_{1}-k_{2}}
\end{aligned}
$$

holds for any natural number $n \geq N_{3}$. As in the proof of Proposition 4.1, one can easily get that $T_{e^{i k_{1} \theta} r^{m}} T_{e^{i k_{2} \theta} \varphi}-T_{e^{i\left(k_{1}+k_{2}\right)} \psi}$ has finite rank if and only if (5.1) holds for $n \geq N_{2}$ and (5.2) holds for $n \geq N_{3}$. Therefore,

$$
\begin{aligned}
& \operatorname{Ran}\left(T_{e^{i k_{1} \theta} \varphi_{1}} T_{e^{i k_{2} \theta} \varphi_{2}}-T_{e^{i\left(k_{1}+k_{2}\right) \theta} \psi}\right) \\
& =\operatorname{span}\left\{\left(T_{e^{i k_{1} \theta} \varphi_{1}} T_{e^{i k_{2} \theta} \varphi_{2}}-T_{e^{i\left(k_{1}+k_{2}\right) \theta} \psi}\right)\left(r^{|l|} e^{i l \theta}\right): l \in \mathbb{Z},-N_{3}+1 \leq l \leq N_{2}-1\right\} \\
& \subseteq \operatorname{span}\left\{r^{|k|} e^{i k \theta}: k \in \Lambda_{2}\right\} .
\end{aligned}
$$

As a direct consequence of Lemma 3.4 then we get (5.3) holds. Moreover, by simple calculations, one can get

$$
N_{2}+N_{3}=\max \left\{\left|k_{1}\right|,\left|k_{2}\right|,\left|k_{1}+k_{2}\right|\right\},
$$

as desired. This completes the proof.

Remark 5.2. Combining (2.2) with the use of adjoint operators, it follows from (5.3) that

$$
\begin{aligned}
& T_{e^{i k_{2} \theta} \varphi_{2}} T_{e^{i k_{1} \theta} \varphi_{1}}-T_{e^{i\left(k_{1}+k_{2}\right) \theta} \psi}=\sum_{j=1}^{N} C_{k_{(j)}}\left(r^{\left|-k_{(j)}+k_{1}+k_{2}\right|} e^{i\left(-k_{(j)}+k_{1}+k_{2}\right) \theta}\right) \otimes\left(r^{\left|-k_{(j)}\right|} e^{-i k_{(j)} \theta}\right) ; \\
& T_{e^{-i k_{1} \theta} \overline{\varphi_{1}}} T_{e^{-i k_{2} \theta} \overline{\varphi_{2}}}-T_{e^{-i\left(k_{1}+k_{2}\right) \theta} \bar{\psi}}=\sum_{j=1}^{N} \overline{C_{k_{(j)}}}\left(r^{\left|-k_{(j)}\right|} e^{-i k_{(j)} \theta}\right) \otimes\left(r^{\left|-k_{(j)}+k_{1}+k_{2}\right|} e^{i\left(-k_{(j)}+k_{1}+k_{2}\right) \theta}\right) ; \\
& T_{e^{-i k_{2} \theta} \overline{\varphi_{2}}} T_{e^{-i k_{1} \theta} \overline{\varphi_{1}}}-T_{e^{-i\left(k_{1}+k_{2}\right) \theta} \bar{\psi}}=\sum_{j=1}^{N} \overline{C_{k_{(j)}}}\left(r^{\left|k_{(j)}-k_{1}-k_{2}\right|} e^{i\left(k_{(j)}-k_{1}-k_{2}\right) \theta}\right) \otimes\left(r^{\left|k_{(j)}\right|} e^{i k_{(j)} \theta}\right) .
\end{aligned}
$$


Furthermore, one can get

$$
\begin{aligned}
& {\left[T_{e^{i k_{1} \theta} \varphi_{1}}, T_{e^{i k_{2} \theta} \varphi_{2}}\right]=}\left(T_{e^{i k_{1} \theta} \varphi_{1}} T_{e^{i k_{2} \theta} \varphi_{2}}-T_{e^{i\left(k_{1}+k_{2}\right) \theta} \psi}\right)-\left(T_{e^{i k_{2} \theta} \varphi_{2}} T_{e^{i k_{1} \theta} \varphi_{1}}-T_{e^{i\left(k_{1}+k_{2}\right) \theta} \psi}\right) \\
&=\sum_{j=1}^{N} C_{k_{(j)}}\left[\left(r^{\left|k_{(j)}\right|} e^{i k_{(j)} \theta}\right) \otimes\left(r^{\left|k_{(j)}-k_{1}-k_{2}\right|} e^{i\left(k_{(j)}-k_{1}-k_{2}\right) \theta}\right)\right. \\
&\left.-\left(r^{\left|-k_{(j)}+k_{1}+k_{2}\right|} e^{i\left(-k_{(j)}+k_{1}+k_{2}\right) \theta}\right) \otimes\left(r^{\left|-k_{(j)}\right|} e^{-i k_{(j)} \theta}\right)\right],
\end{aligned}
$$

which represents in the same form as (4.3). But the above form is not necessarily the canonical form, as here $k_{(i)}=-k_{(j)}+k_{1}+k_{2}$ may hold for some $k_{(i)}, k_{(j)} \in \Lambda_{2}$. Thus, the rank of $\left[T_{e^{i k_{1} \theta} \varphi_{1}}, T_{e^{i k_{2} \theta} \varphi_{2}}\right]$ should be less or equal to $2 \operatorname{rank}\left(T_{e^{i k_{1} \theta} \varphi_{1}} T_{e^{i k_{2} \theta} \varphi_{2}}-T_{e^{i\left(k_{1}+k_{2}\right) \theta} \psi}\right)$, which corresponds to Corollary 2.3. In fact, Example 4.5 and Example 5.5 also illustrate this point.

The following corollary gives a complete description of the finite rank generalized semicommutators of quasihomogeneous Toeplitz operators with some special degrees.

Corollary 5.3. Let $k \in \mathbb{Z}$, and let $\varphi_{1}, \varphi_{2}$ and $\psi$ be radial T-functions. Then on $L_{h}^{2}$, the following statements hold.

(a) If $\varphi_{1}$ is nonconstant and $\varphi_{2} \neq 0$, then the generalized semicommutator $T_{\varphi_{1}} T_{e^{i k \theta} \varphi_{2}}-$ $T_{e^{i k \theta} \psi}$ has finite rank if and only if $k=0$ and $\psi$ is a solution of the equation

$$
\mathbb{I} *_{M} \psi=\varphi_{1} *_{M} \varphi_{2}
$$

(b) If $k \neq 0$, then the generalized semicommutator $T_{e^{i k \theta} \varphi_{1}} T_{e^{-i k \theta} \varphi_{2}}-T_{\psi}$ has finite rank if and only if $|k|=1, \varphi_{1} *_{M} \varphi_{2}=C\left(r *_{M} r^{-1}\right)$ and $\psi=C$ for some constant $C$.

In each case, the rank of the generalized semicommutator is zero.

Proof. First assume $T_{\varphi_{1}} T_{e^{i k \theta} \varphi_{2}}-T_{e^{i k \theta} \psi}$ has finite rank, then combining Corollary 2.3 with Corollary 4.3 we get $k=0$. Thus Proposition 5.1 gives that $\operatorname{rank}\left(T_{\varphi_{1}} T_{e^{i k \theta} \varphi_{2}}-T_{e^{i k \theta} \psi}\right) \leq 0$, and so $T_{\varphi_{1}} T_{e^{i k \theta} \varphi_{2}}=T_{e^{i k \theta} \psi}$. Then by [21, Corollary 3.1], the above equation holds if and only if $\psi$ is a solution of the equation $\mathbb{I} *_{M} \psi=\varphi_{1} *_{M} \varphi_{2}$, and hence condition (a) holds.

The proof of (b) is similar. This completes the proof.

Next, we give our main result in this section, which completely characterize when the generalized semicommutator $T_{e^{i k_{1} \theta} r^{m}} T_{e^{i k_{2} \theta} \varphi}-T_{e^{i\left(k_{1}+k_{2}\right) \theta} \psi}$ to be finite rank.

Theorem 5.4. Let $k_{1}, k_{2} \in \mathbb{Z}$, and let $m \in \mathbb{R}, m \geq-1$. Then for nonzero radial T-functions $\varphi$ and $\psi$ on $D$, the generalized semicommutator $T_{e^{i k_{1}{ }^{\theta} r^{m}}} T_{e^{i k_{2} \theta} \varphi}-T_{e^{i\left(k_{1}+k_{2}\right) \theta} \psi}$ has finite rank on $L_{h}^{2}$ if and only if one of the following conditions holds:

(1) $k_{1}=m=0$ and $\psi=\varphi$.

(2) $k_{2}=0, \varphi=C$ and $\psi=C r^{m}$ for some constant $C$.

(3) $k_{1}=k_{2}=0$ and $\psi(r)=\varphi(r)-m r^{m} \int_{r}^{1} \frac{\varphi(t)}{t^{m+1}} d t$.

(4) $k_{1} k_{2}=-1, \varphi=C\left(\frac{m+1}{2} r^{-1}-\frac{m-1}{2} r\right)$ and $\psi=C$ for some constant $C$.

(5) $k_{1} k_{2}<-1,\left|k_{2}\right| \geq 2, m+\left|k_{1}\right|=0, \varphi(r)=C r^{\left|k_{2}\right|}$ and $\psi=C r^{\left|k_{2}\right|-1}$.

(6) $k_{1} k_{2}<-1,\left|k_{2}\right|=1, \widehat{\varphi}(z)=C \frac{\Gamma\left(\frac{z-1}{2 \mid k_{1}}\right) \Gamma\left(\frac{z+m+\left|k_{1}\right|+1}{2\left|k_{1}\right|}\right)}{\Gamma\left(\frac{z+2\left|k_{1}\right|+1}{2\left|k_{1}\right|}\right) \Gamma\left(\frac{z+m+\left|k_{1}\right|-1}{2\left|k_{1}\right|}\right)}$ and

$$
\widehat{\psi}(z)=C \frac{\Gamma\left(\frac{z+\left|k_{1}\right|-1}{2\left|k_{1}\right|}\right) \Gamma\left(\frac{z+m+1}{2\left|k_{1}\right|}\right)}{\Gamma\left(\frac{z+\left|k_{1}\right|+1}{2\left|k_{1}\right|}\right) \Gamma\left(\frac{z+m+2\left|k_{1}\right|-1}{2\left|k_{1}\right|}\right)} .
$$

(7) $k_{1} k_{2}>0,\left|k_{2}\right|<m+2, \widehat{\varphi}(z)=C \frac{\Gamma\left(\frac{z+\left|k_{2}\right|}{2\left|k_{1}\right|}\right) \Gamma\left(\frac{z+m+\left|k_{1}\right|-\left|k_{2}\right|}{2\left|k_{1}\right|}\right)}{\Gamma\left(\frac{z+2\left|k_{1}\right|-\left|k_{2}\right|}{2\left|k_{1}\right|}\right) \Gamma\left(\frac{z+m+\left|k_{1}\right|+\left|k_{2}\right|}{2\left|k_{1}\right|}\right)}$ and

$$
\widehat{\psi}(z)=C \frac{\Gamma\left(\frac{z+\left|k_{1}\right|+\left|k_{2}\right|}{2\left|k_{1}\right|}\right) \Gamma\left(\frac{z+m-\left|k_{2}\right|}{2\left|k_{1}\right|}\right)}{\Gamma\left(\frac{z+\left|k_{1}\right|-\left|k_{2}\right|}{2\left|k_{1}\right|}\right) \Gamma\left(\frac{z+m+2\left|k_{1}\right|+\left|k_{2}\right|}{2\left|k_{1}\right|}\right)} .
$$


(8) $k_{1} k_{2}>0,\left|k_{2}\right| \geq m+2, m=(2 n+1)\left|k_{1}\right|$ for some $n \in \mathbb{N}$,

$$
\widehat{\varphi}(z)=C \frac{\left(\frac{z-\left|k_{2}\right|}{2\left|k_{1}\right|}+1\right)\left(\frac{z-\left|k_{2}\right|}{2\left|k_{1}\right|}+2\right) \cdots\left(\frac{z-\left|k_{2}\right|}{2\left|k_{1}\right|}+n\right)}{\frac{z+\left|k_{2}\right|}{2\left|k_{1}\right|}\left(\frac{z+\left|k_{2}\right|}{2\left|k_{1}\right|}+1\right) \cdots\left(\frac{z+\left|k_{2}\right|}{2\left|k_{1}\right|}+n\right)}
$$

and

$$
\widehat{\psi}(z)=C \frac{\frac{z+\left|k_{1}\right|-\left|k_{2}\right|}{2\left|k_{1}\right|}\left(\frac{z+\left|k_{1}\right|-\left|k_{2}\right|}{2\left|k_{1}\right|}+1\right) \cdots\left(\frac{z+\left|k_{1}\right|-\left|k_{2}\right|}{2\left|k_{1}\right|}+n-1\right)}{\frac{z+\left|k_{1}\right|+\left|k_{2}\right|}{2\left|k_{1}\right|}\left(\frac{z+\left|k_{1}\right|+\left|k_{2}\right|}{2\left|k_{1}\right|}+1\right) \cdots\left(\frac{z+\left|k_{1}\right|+\left|k_{2}\right|}{2\left|k_{1}\right|}+n\right)} .
$$

In each condition (1)-(4), rank $\left(T_{e^{i k_{1} \theta} r_{m}} T_{e^{i k_{2} \theta} \varphi}-T_{e^{i\left(k_{1}+k_{2}\right) \theta} \psi}\right)=0$. In each condition (5)-(8),

$$
\operatorname{Ran}\left(T_{e^{i k_{1} \theta} r^{m}} T_{e^{i k_{2} \theta} \varphi}-T_{e^{i\left(k_{1}+k_{2}\right) \theta} \psi}\right)=\operatorname{span}\left\{r^{|k|} e^{i k \theta}: k \in \Lambda_{2}\right\}
$$

and

$$
T_{e^{i k_{1} \theta} r^{m}} T_{e^{i k_{2} \theta} \varphi}-T_{e^{i\left(k_{1}+k_{2}\right) \theta} \psi}=\sum_{k \in \Lambda_{2}} C_{k}\left(r^{|k|} e^{i k \theta}\right) \otimes\left(r^{\left|k-k_{1}-k_{2}\right|} e^{i\left(k-k_{1}-k_{2}\right) \theta}\right)
$$

for some nonzero constant $C_{k}$. Therefore,

$$
\operatorname{rank}\left(T_{e^{i k_{1}{ }^{\theta} r^{m}}} T_{e^{i k_{2} \theta} \varphi}-T_{e^{i\left(k_{1}+k_{2}\right) \theta} \psi}\right)=\max \left\{\left|k_{1}\right|-1,\left|k_{2}\right|-1,\left|k_{1}+k_{2}\right|-1\right\} .
$$

Proof. First we suppose $k_{1} k_{2}=0$, or $k_{1} k_{2}=-1$. Then Corollary[5.3] shows that $T_{e^{i k_{1} \theta} r^{m}} T_{e^{i k_{2} \theta} \varphi}-$ $T_{e^{i\left(k_{1}+k_{2}\right) \theta} \psi}$ has finite rank if and only if one of the conditions (1)-(4) holds. Moreover, in each condition, 21. Corollary 3.1] shows that $T_{e^{i k_{1} \theta} r^{m}} T_{e^{i k_{2} \theta} \varphi}=T_{e^{i\left(k_{1}+k_{2}\right) \theta} \psi}$, as desired.

Next, we suppose $k_{1} k_{2} \neq 0$ and $k_{1} k_{2} \neq-1$. Combining Theorem 2.2 with the use of adjoint operators, we can further assume $k_{1}>0$. Then by Proposition [5.1, $T_{e^{i k_{1} \theta} r^{m}} T_{e^{i k_{2} \theta} \varphi}-T_{e^{i\left(k_{1}+k_{2}\right) \theta} \psi}$ has finite rank if and only if

$$
2\left(n+k_{2}+1\right) \widehat{r^{m}}\left(2 n+k_{1}+2 k_{2}+2\right) \widehat{\varphi}\left(2 n+k_{2}+2\right)=\widehat{\psi}\left(2 n+k_{1}+k_{2}+2\right)
$$

holds for all $n \geq N_{2}=\max \left\{0,-k_{2},\right\}$, and

$$
2\left(n-k_{2}+1\right) \widehat{r^{m}}\left(2 n-k_{1}-2 k_{2}+2\right) \widehat{\varphi}\left(2 n-k_{2}+2\right)=\widehat{\psi}\left(2 n-k_{1}-k_{2}+2\right)
$$

holds for all $n \geq N_{3}=\max \left\{0, k_{1}+k_{2}\right\}$.

Now, we assume $T_{e^{i k_{1} \theta} r^{m}} T_{e^{i k_{2} \theta} \varphi}-T_{e^{i\left(k_{1}+k_{2}\right) \theta} \psi}$ has finite rank. Then by Corollary 2.3 the commutator $\left[T_{e^{i k_{1} \theta} r^{m}}, T_{e^{i k_{2} \theta} \varphi}\right]$ has finite rank, and hence one of the conditions (4) and (6)-(9) of Theorem 4.4 holds. On the other hand, (5.4) implies

$$
\widehat{\psi}(z)=\left(z-k_{1}+k_{2}\right) \widehat{r^{m}}\left(z+k_{2}\right) \widehat{\varphi}\left(z-k_{1}\right) .
$$

Thus, by (4.9) we get

$$
\widehat{\psi}(z)=C \frac{z-k_{1}+k_{2}}{z+m+k_{2}} \frac{\Gamma\left(\frac{z+k_{2}-k_{1}}{2 k_{1}}\right) \Gamma\left(\frac{z+m-k_{2}}{2 k_{1}}\right)}{\Gamma\left(\frac{z+k_{1}-k_{2}}{2 k_{1}}\right) \Gamma\left(\frac{z+m+k_{2}}{2 k_{1}}\right)}=C \frac{\Gamma\left(\frac{z+k_{1}+k_{2}}{2 k_{1}}\right) \Gamma\left(\frac{z+m-k_{2}}{2 k_{1}}\right)}{\Gamma\left(\frac{z+k_{1}-k_{2}}{2 k_{1}}\right) \Gamma\left(\frac{z+m+2 k_{1}+k_{2}}{2 k_{1}}\right)} .
$$

As in the proof of Lemma 3.7, it is easy to verify that $\psi(r)$ is a T-function if and only if one of the following conditions holds:

- $k_{2} \leq-k_{1}-2$ and $m+k_{1}=0$.

- $-k_{1}-2<k_{2}<m+2$.

- $k_{2} \geq m+2$ and $m=(2 n+1) k_{1}$ for some $n \in \mathbb{N}$.

Then combining with Theorem 4.4 it is easy to show that one of the conditions (5)-(8) holds.

Conversely, if one of the conditions (5)-(8) holds, then (4.8) and (5.4) hold for all $n \geq N_{1}=$ $N_{2}=\max \left\{0,-k_{2},\right\}$. From (4.8) and (5.4) we can deduce

$$
2\left(n+k_{1}+1\right) \widehat{r^{m}}\left(2 n+k_{1}+2\right) \widehat{\varphi}\left(2 n+2 k_{1}+k_{2}+2\right)=\widehat{\psi}\left(2 n+k_{1}+k_{2}+2\right)
$$

holds for all $n \geq \max \left\{0,-k_{2},\right\}$. Replacing $n$ by $n-k_{1}-k_{2}$, then the above equation implies that (5.5) holds for all $n \geq \max \left\{k_{1}, k_{1}+k_{2},\right\}$. As in the proof of Proposition 4.1, we get (5.5) holds for all $n \geq N_{3}=\max \left\{0, k_{1}+k_{2}\right\}$, and hence $T_{e^{i k_{1} \theta} r^{m}} T_{e^{i k_{2} \theta} \varphi}-T_{e^{i\left(k_{1}+k_{2}\right) \theta} \psi}$ has finite rank. 
According to Proposition 5.1, to finish the proof we only need to show that, for any $l \in$ $\left\{-N_{3}+1, \cdots, N_{2}-1\right\},\left(T_{e^{i k_{1} \theta} r^{m}} T_{e^{i k_{2} \theta} \varphi}-T_{e^{i\left(k_{1}+k_{2}\right)} \psi}\right)\left(r^{|l|} e^{i l \theta}\right)=C_{l} r^{\left|l+k_{1}+k_{2}\right|} e^{i\left(l+k_{1}+k_{2}\right) \theta}$ must hold for some nonzero constant $C_{l}$. To show this we need to discuss three cases.

Case 1. Suppose $k_{1}=-m=1, k_{2} \leq-2$. Thus $N_{2}=-k_{2}$ and $N_{3}=0$. So for any $0<n \leq-k_{2}-1$, by Lemma 3.1 we get

$$
\begin{aligned}
& \left(T_{e^{i k_{1} \theta} r^{m}} T_{e^{i k_{2} \theta} \varphi}-T_{e^{i\left(k_{1}+k_{2}\right) \theta} \psi}\right)\left(z^{n}\right)=0 \\
& \Longleftrightarrow 2\left(-k_{2}-n+1\right) r^{m}\left(-k_{1}-2 k_{2}-2 n+2\right) \widehat{\varphi}\left(-k_{2}+2\right)=\widehat{\psi}\left(-k_{1}-k_{2}+2\right) .
\end{aligned}
$$

Denote

$$
\begin{aligned}
H(x) & =2\left(-k_{2}-x+1\right) \widehat{r^{m}}\left(-k_{1}-2 k_{2}-2 x+2\right) \\
& =\frac{2\left(-k_{2}-x+1\right)}{m-k_{1}-2 k_{2}-2 x+2}=\frac{1}{1-\frac{1}{-k_{2}-x+1}},
\end{aligned}
$$

which implies that $H(x)$ is strictly monotone increasing on $\left[0,-k_{2}\right)$. Hence

$$
H(0)<H(1)<\cdots<H\left(-k_{2}-1\right) .
$$

On the other hand, by taking $n=0$ in (5.5), we obtain

$$
H(0) \widehat{\varphi}\left(-k_{2}+2\right)=\widehat{\psi}\left(-k_{1}-k_{2}+2\right),
$$

Since $\widehat{\varphi}\left(-k_{2}+2\right) \neq 0$, it follows that (5.6) would not hold, as desired.

Case 2. Suppose $k_{1}>0$ and $k_{2}=-1$. Thus $N_{2}=1$ and $N_{3}=k_{1}+k_{2}$. So for any $0 \leq n<k_{1}+k_{2}$, by Lemma 3.1 we get

$$
\begin{aligned}
& \left(T_{e^{i k_{1} \theta} r^{m}} T_{e^{i k_{2} \theta} \varphi}-T_{e^{i\left(k_{1}+k_{2}\right) \theta} \psi}\right)\left(\bar{z}^{n}\right)=0 \\
& \Longleftrightarrow 2\left(n-k_{2}+1\right) \widehat{r}^{m}\left(k_{1}+2\right) \widehat{\varphi}\left(2 n-k_{2}+2\right)=\widehat{\psi}\left(k_{1}+k_{2}+2\right) \\
& \Longleftrightarrow F(n) \widehat{r^{m}}\left(k_{1}+2\right)=\widehat{\psi}\left(k_{1}+k_{2}+2\right),
\end{aligned}
$$

where $F(x)$ is defined by (4.11). Since $a=\frac{k_{2}}{k_{1}}<0$ and $b=\frac{m+k_{1}}{2 k_{1}}>0$, it follows that $F(x)$ is strictly monotone decreasing on $(-1,+\infty)$. Thus,

$$
F(0)>F(1)>\cdots>F\left(k_{1}+k_{2}\right) .
$$

On the other hand, by taking $n=k_{1}+k_{2}$ in (5.5), we obtain

$$
F\left(k_{1}+k_{2}\right) \widehat{r^{m}}\left(k_{1}+2\right)=\widehat{\psi}\left(k_{1}+k_{2}+2\right)
$$

and hence (5.7) would not hold.

Case 3. Suppose $k_{1} k_{2}>0$. Moreover, both condition (7) and condition (8) imply that $m+k_{1} \neq 0$. Thus $N_{2}=0$ and $N_{3}=k_{1}+k_{2}$.

First, for any $1 \leq n \leq k_{2}$, by Lemma 3.1 we get

$$
\begin{aligned}
& \left(T_{e^{i k_{1} \theta} r^{m}} T_{e^{i k_{2} \theta} \varphi}-T_{e^{i\left(k_{1}+k_{2}\right) \theta} \psi}\right)\left(\bar{z}^{n}\right)=0 \\
& \Longleftrightarrow 2\left(k_{2}-n+1\right) \widehat{r^{m}}\left(k_{1}+2 k_{2}-2 n+2\right) \widehat{\varphi}\left(k_{2}+2\right)=\widehat{\psi}\left(k_{1}+k_{2}+2\right) .
\end{aligned}
$$

Denote

$$
\begin{aligned}
P(x) & =2\left(k_{2}-x+1\right) \widehat{r^{m}}\left(k_{1}+2 k_{2}-2 x+2\right) \\
& =\frac{2\left(k_{2}-x+1\right)}{m+k_{1}+2 k_{2}-2 x+2}=\frac{1}{\frac{m+k_{1}}{2\left(k_{2}-x+1\right)}+1},
\end{aligned}
$$

which implies that $P(x)$ is strictly monotone increasing on $[0,+\infty)$. Hence

$$
P(0)<P(1)<\cdots<P\left(k_{2}\right) .
$$

On the other hand, by taking $n=0$ in (5.4), we obtain

$$
P(0) \widehat{\varphi}\left(k_{2}+2\right)=\widehat{\psi}\left(k_{1}+k_{2}+2\right) .
$$

Since $\widehat{\varphi}\left(k_{2}+2\right) \neq 0$, it follows that (5.8) would not hold. 
Next, for any $k_{2} \leq n<k_{1}+k_{2}$, by Lemma 3.1 we get

$$
\begin{aligned}
& \left(T_{e^{i k_{1} \theta} r^{m}} T_{e^{i k_{2} \theta} \varphi}-T_{e^{i\left(k_{1}+k_{2}\right) \theta} \psi}\right)\left(\bar{z}^{n}\right)=0 \\
& \Longleftrightarrow 2\left(n-k_{2}+1\right) \widehat{r^{m}}\left(k_{1}+2\right) \widehat{\varphi}\left(2 n-k_{2}+2\right)=\widehat{\psi}\left(k_{1}+k_{2}+2\right) \\
& \Longleftrightarrow F(n) \widehat{r^{m}}\left(k_{1}+2\right)=\widehat{\psi}\left(k_{1}+k_{2}+2\right) .
\end{aligned}
$$

Since $a=\frac{k_{2}}{k_{1}}>0$ and $b=\frac{m+k_{1}}{2 k_{1}}>0$, it follows that $F(x)$ is strictly monotone increasing on $\left(k_{2}-1,+\infty\right)$. Thus,

$$
F\left(k_{2}\right)<F\left(k_{2}+1\right)<\cdots<F\left(k_{1}+k_{2}\right) .
$$

On the other hand, by taking $n=k_{1}+k_{2}$ in (5.5), we obtain

$$
F\left(k_{1}+k_{2}\right) \widehat{r^{m}}\left(k_{1}+2\right)=\widehat{\psi}\left(k_{1}+k_{2}+2\right),
$$

and hence (5.9) would not hold, as desired. This completes the proof.

Below we present the examples of nonzero generalized semicommutators of finite rank, corresponding to the conditions (5)-(8) of Theorem 5.4.

Example 5.5. Let $\varphi(r)$ be a nonzero radial T-function on $D$, then on $L_{h}^{2}$, the following statements hold.

(1) $T_{e^{i \theta} r^{-1}} T_{e^{-3 i \theta} \varphi(r)}-T_{e^{-2 i \theta} \psi}$ has finite rank if and only if

$$
\varphi(r)=C r^{3} \text { and } \psi(r)=C r^{2} .
$$

In this case, $T_{e^{i \theta} r^{-1}} T_{e^{-3 i \theta} \varphi(r)}-T_{e^{-2 i \theta} \psi}=C\left[\frac{1}{2}\left(1 \otimes z^{2}\right)+\frac{1}{6}(\bar{z} \otimes z)\right]$.

(2) $T_{e^{2 i \theta} r^{6}} T_{e^{-i \theta} \varphi(r)}-T_{e^{i \theta} \psi}$ has finite rank if and only if

$$
\varphi(r)=C\left(\frac{3}{r}-r^{3}\right) \text { and } \psi(r)=C\left(r+r^{5}\right) .
$$

In this case, $T_{e^{2 i \theta} r^{6}} T_{e^{-i \theta} \varphi(r)}-T_{e^{i \theta} \psi}=\frac{19 C}{30}(z \otimes 1)$.

(3) Let $m \in \mathbb{R}, m \geq-1$. Then $T_{e^{i \theta} r^{m}} T_{e^{2 i \theta} \varphi(r)}-T_{e^{3 i \theta} \psi}$ has finite rank if and only if $m>0$,

$$
\begin{gathered}
\varphi(r)=C\left[(m+1) r^{m+1}-(m-1) r^{m-1}\right] \text { and } \\
\psi(r)=\frac{C}{4}\left[(m-3)(m-1) r^{m-2}-(m-1)(m+1) r^{m}+(m+1)(m+3) r^{m+2}\right] .
\end{gathered}
$$

In this case, $T_{e^{i \theta} r^{m}} T_{e^{2 i \theta} \varphi(r)}-T_{e^{3 i \theta} \psi}=-\frac{192(m+1) C}{(m+7)(m+5)(m+3)}\left[\frac{2}{m+3}\left(z \otimes \bar{z}^{2}\right)+\frac{1}{m+5}\left(z^{2} \otimes \bar{z}\right)\right]$.

(4) $T_{e^{i \theta} r^{3}} T_{e^{6 i \theta} \varphi(r)}-T_{e^{7 i \theta} \psi}$ has finite rank if and only if

$$
\varphi(r)=C\left[6 r^{8}-5 r^{6}\right] \text { and } \psi(r)=C\left[7 r^{9}-6 r^{7}\right] .
$$

In this case,

$$
\begin{aligned}
T_{e^{i \theta} r^{3}} T_{e^{6 i \theta} \varphi(r)}-T_{e^{7 i \theta} \psi}=C[ & -\frac{2}{9}\left(z \otimes \bar{z}^{6}\right)-\frac{1}{72}\left(z^{6} \otimes \bar{z}\right)-\frac{5}{28}\left(z^{2} \otimes \bar{z}^{5}\right) \\
& \left.-\frac{2}{49}\left(z^{5} \otimes \bar{z}^{2}\right)-\frac{8}{63}\left(z^{3} \otimes \bar{z}^{4}\right)-\frac{5}{63}\left(z^{4} \otimes \bar{z}^{3}\right)\right] .
\end{aligned}
$$

Finally, we show some interesting applications of Theorem 5.4 .

Corollary 5.6. Let $k_{1}, k_{2} \in \mathbb{Z}$ such that $k_{1}>0$ or $k_{1}=-1$, and let $\varphi(r), \psi(r)$ be two nonzero radial T-functions on $D$. Then on $L_{h}^{2}$, the following statements hold.

(a) $T_{e^{i k_{1} \theta} r^{k_{1}}} T_{e^{i k_{2} \theta} \varphi(r)}-T_{e^{i\left(k_{1}+k_{2}\right) \theta} \psi}$ has finite rank if and only if

$$
k_{2}>\max \left\{-2,-k_{1}-2\right\}, \quad \varphi(r)=C r^{k_{2}} \text { and } \psi(r)=C r^{k_{1}+k_{2}} .
$$

(b) $T_{e^{-i k_{1} \theta} r^{k_{1}}} T_{e^{i k_{2} \theta} \varphi(r)}-T_{e^{i\left(-k_{1}+k_{2}\right) \theta} \psi}$ has finite rank if and only if

$$
k_{2}<\min \left\{2, k_{1}+2\right\}, \quad \varphi(r)=C r^{-k_{2}} \text { and } \psi(r)=C r^{k_{1}-k_{2}} .
$$


Proof. If $k_{1}>0$, then by Theorem 5.4 $T_{e^{i k_{1} \theta} r^{k_{1}}} T_{e^{i k_{2} \theta} \varphi(r)}-T_{e^{i\left(k_{1}+k_{2}\right) \theta} \psi}$ has finite rank if and only if $k_{2}$ satisfies one of the conditions (2), (4) and (6)-(8) and

$$
\widehat{\varphi}(z)=\frac{C}{z+k_{2}} \text { and } \widehat{\psi}(z)=\frac{C}{z+k_{1}+k_{2}} .
$$

In other words, $k_{2}>-2, \varphi(r)=C r^{k_{2}}$ and $\psi(r)=C r^{k_{1}+k_{2}}$.

Similarly, if $k_{1}=-1$, then $T_{e^{i k_{1} \theta} r^{k_{1}}} T_{e^{i k_{2} \theta} \varphi(r)}-T_{e^{i\left(k_{1}+k_{2}\right) \theta} \psi}$ has finite rank if and only if one of the conditions (2), (4), (5) and (7) holds, which implies $k_{2}>-k_{1}-2, \varphi(r)=C r^{k_{2}}$ and $\psi(r)=C r^{k_{1}+k_{2}}$, as desired.

Condition (a) together with Theorem 2.2 and the use of adjoint operators yields that condition (b) holds. This completes the proof.

Corollary 5.7. Let $k_{1}, k_{2} \in \mathbb{Z}$ such that $k_{1} k_{2} \neq 0$, and let $m_{1}, m_{2} \in \mathbb{R}$ such that greater than or equal to -1. Then $T_{e^{i k_{1} \theta} r^{m_{1}}} T_{e^{i k_{2} \theta} r^{m_{2}}}-T_{e^{i\left(k_{1}+k_{2}\right) \theta} \psi}$ has finite rank on $L_{h}^{2}$ if and only if one of the following conditions holds:

(1) $k_{1}=k_{2}, m_{1}=m_{2},\left|k_{1}\right|<m_{1}+2$ and $\psi(r)=\frac{m_{1}+\left|k_{1}\right|}{2\left|k_{1}\right|} r^{m_{1}+\left|k_{1}\right|}-\frac{m_{1}-\left|k_{1}\right|}{2\left|k_{1}\right|} r^{m_{1}-\left|k_{1}\right|}$.

(2) $k_{1}=m_{1}, k_{2}=m_{2}, k_{1}+k_{2} \neq-2$ and $\psi(r)=r^{k_{1}+k_{2}}$.

(3) $k_{1}=-m_{1}, k_{2}=-m_{2}, k_{1}+k_{2} \neq 2$ and $\psi(r)=r^{-k_{1}-k_{2}}$.

Proof. First suppose $T_{e^{i k_{1} \theta} r^{m_{1}}} T_{e^{i k_{2} \theta} r^{m_{2}}}-T_{e^{i\left(k_{1}+k_{2}\right) \theta} \psi}$ has finite rank. Then by Corollary 2.3 and Corollary 4.7, one of the following cases holds.

Case 1. $k_{1}=k_{2}$ and $m_{1}=m_{2}$. Since $k_{1} k_{2} \neq 0$, we get that condition (7) of Theorem 5.4 is the only one which satisfies this case, and hence $\left|k_{1}\right|<m_{1}+2$. Notice that $\varphi(r)=r^{m_{1}}$, then we get

$$
\widehat{\psi}(z)=\frac{z}{\left(z+m_{1}-\left|k_{1}\right|\right)\left(z+m_{1}+\left|k_{1}\right|\right)}=\frac{m_{1}+\left|k_{1}\right|}{2\left|k_{1}\right|} \frac{1}{z+m_{1}+\left|k_{1}\right|}-\frac{m_{1}-\left|k_{1}\right|}{2\left|k_{1}\right|} \frac{1}{z+m_{1}-\left|k_{1}\right|},
$$

which implies $\psi(r)=\frac{m_{1}+\left|k_{1}\right|}{2\left|k_{1}\right|} r^{m_{1}+\left|k_{1}\right|}-\frac{m_{1}-\left|k_{1}\right|}{2\left|k_{1}\right|} r^{m_{1}-\left|k_{1}\right|}$.

Case 2. $k_{1}=m_{1}, k_{2}=m_{2}$. Notice that $m_{1} \geq-1, m_{2} \geq-1$ and $k_{1} k_{2} \neq 0$, then

$$
k_{2}>\max \left\{-2,-k_{1}-2\right\} \Longleftrightarrow k_{1}+k_{2} \neq-2 \text {. }
$$

So by condition (a) of Corollary 5.6. we get $k_{1}+k_{2} \neq-2$ and $\psi(r)=r^{k_{1}+k_{2}}$.

Case 3. $k_{1}=-m_{1}, k_{2}=-m_{2}$. Then $T_{e^{i k_{1} \theta} r^{m_{1}}} T_{e^{i k_{2} \theta} r^{m_{2}}}-T_{e^{i\left(k_{1}+k_{2}\right) \theta} \psi}$ can be written as $T_{e^{-i\left(-k_{1}\right) \theta} r^{-k_{1}}} T_{e^{i k_{2} \theta} r^{-k_{2}}}-T_{e^{i\left(k_{1}+k_{2}\right) \theta} \psi}$. Since $k_{1} \leq 1, k_{2} \leq 1$, we get

$$
k_{2}<\min \left\{2,-k_{1}+2\right\} \Longleftrightarrow k_{1}+k_{2} \neq 2 .
$$

So by condition (b) of Corollary [5.6, we get $k_{1}+k_{2} \neq 2$ and $\psi(r)=r^{-k_{1}-k_{2}}$.

By condition (7) of Theorem 5.4 and Corollary [5.6 the converse implication is clear. This completes the proof.

Corollary 5.8. Let $k_{1}, k_{2} \in \mathbb{Z}$ such that $k_{1} k_{2} \neq 0$, and let $m \in \mathbb{R}, m \geq-1$. Then for $a$ nonzero radial T-function $\varphi(r)$ on $D$, the semicommutator $\left(T_{e^{i k_{1} \theta} r^{m}}, T_{e^{i k_{2} \theta} \varphi}\right]$ has finite rank on $L_{h}^{2}$ if and only if one of the following conditions holds:

(1) $m=k_{1}, k_{2}>\max \left\{-2,-k_{1}-2\right\}$ and $\varphi(r)=r^{k_{2}}$.

(2) $m=-k_{1}, k_{2}<\min \left\{2,-k_{1}+2\right\}$ and $\varphi(r)=r^{-k_{2}}$.

Proof. If the semicommutator $\left(T_{e^{i k_{1} \theta} r^{m}}, T_{e^{i k_{2} \theta} \varphi}\right]$ has finite rank, then it follows from (5.1) that

$$
\left(z-k_{1}+k_{2}\right) \widehat{r^{m}}\left(z+k_{2}\right) \widehat{\varphi}\left(z-k_{1}\right)=\widehat{r^{m} \varphi}(z),
$$

which is equivalent to $\left(z-k_{1}+k_{2}\right) \widehat{\varphi}\left(z-k_{1}\right)=\left(z+m+k_{2}\right) \widehat{\varphi}(z+m)$. Using the same argument as in the proof of [16, Theorem 6], we can conclude that

$$
\widehat{\varphi}\left(z-k_{1}\right)=\frac{C}{z-k_{1}+k_{2}}
$$

or $m+k_{1}=0$ holds. 
If (5.11) holds, then $\varphi(r)=C r^{k_{2}}$. Notice that $\varphi(r)$ is a T-function, and hence $k_{2} \geq-1$. Then by Corollary 5.7 we get $m=k_{1}$ and $k_{1}+k_{2} \neq-2$. Because of (5.10), we have that condition (1) holds.

If $m+k_{1}=0$, then $\left(T_{e^{i k_{1} \theta} r^{m}}, T_{e^{i k_{2} \theta} \varphi}\right]$ can be written as $T_{e^{-i\left(-k_{1}\right) \theta} r^{-k_{1}}} T_{e^{i k_{2} \theta} \varphi}-T_{e^{i\left(k_{1}+k_{2}\right) \theta} r^{-k_{1}} \varphi}$ Notice that $-k_{1}=m \geq-1$, and $k_{1} k_{2} \neq 0$, then Corollary 5.6 shows that condition (2) holds.

By Corollary [5.6 the converse implication is clear. This completes the proof.

\section{RANKS OF COMmUtators AND SEMicommutators ON $L_{a}^{2}$}

On the Bergman space, Čučković and Louhichi [15] proved that if two quasihomogeneous Toeplitz operators have both positive or both negative degrees and if their commutator (or semicommutator) has finite rank, then the commutator (or semicommutator) must be zero. Moreover, they presented two examples of nonzero finite rank commutators and semicommutators of two quasihomogeneous Toeplitz operators of opposite degrees:

$$
\operatorname{rank}\left(\left[T_{e^{2 i \theta} r^{6}}, T_{e^{-i \theta}\left(\frac{3}{r}-r^{3}\right)}\right]\right)=1 \text { and } \operatorname{rank}\left(\left(T_{e^{i \theta} \frac{1}{r}}, T_{e^{-i \theta} r}\right]\right)=1 .
$$

In this section, we continue this line of investigation and study finite rank commutators and generalized semicommutators of quasihomogeneous Toeplitz operators on the Bergman space, with one of the symbols being of the form $e^{i k \theta} r^{m}$. We will show that the corresponding results are different from those of harmonic Bergman space.

The following lemma is from [25, Lemma 5.3] which we shall use often in this section.

Lemma 6.1. Let $k \in \mathbb{Z}$ and let $\varphi$ be a radial $T$-function. Then on $L_{a}^{2}$, for each $n \in \mathbb{N}$ we have

$$
T_{e^{i k \theta} \varphi}\left(z^{n}\right)= \begin{cases}2(n+k+1) \widehat{\varphi}(2 n+k+2) z^{n+k}, & \text { if } n \geq-k, \\ 0, & \text { if } n<-k .\end{cases}
$$

Unlike harmonic Bergman space case, the following theorem shows that the commutator $\left[T_{e^{i k_{1} \theta} r^{m}}, T_{e^{i k_{2} \theta} \varphi}\right]$ on $L_{a}^{2}$ can only has finite rank 0 or 1 .

Theorem 6.2. Let $k_{1}, k_{2} \in \mathbb{Z}$, and let $m \in \mathbb{R}, m \geq-1$. Then for a nonzero radial $T$-function $\varphi(r)$ on $D$, the commutator $\left[T_{e^{i k_{1} \theta} r^{m}}, T_{e^{i k_{2} \theta} \varphi}\right]$ has finite rank on $L_{a}^{2}$ if and only if one of the following conditions holds:

(1) $k_{1}=m=0$.

(2) $k_{2}=0$ and $\varphi=C$.

(3) $k_{1}=k_{2}=0$.

(4) $k_{1} k_{2}>0,\left|k_{2}\right|<m+\left|k_{1}\right|+2$ and $\widehat{\varphi}(z)=C \frac{\Gamma\left(\frac{z+\left|k_{2}\right|}{2\left|k_{1}\right|}\right) \Gamma\left(\frac{z+m+\left|k_{1}\right|-\left|k_{2}\right|}{2\left|k_{1}\right|}\right)}{\Gamma\left(\frac{z+2\left|k_{1}\right|-\left|k_{2}\right|}{2\left|k_{1}\right|}\right) \Gamma\left(\frac{z+m+\left|k_{1}\right|+\left|k_{2}\right|}{2\left|k_{1}\right|}\right)}$.

(5) $k_{1} k_{2}>0,\left|k_{2}\right| \geq m+\left|k_{1}\right|+2, m=(2 n+1)\left|k_{1}\right|$ for some $n \in \mathbb{N}$ and

$$
\widehat{\varphi}(z)=C \frac{\left(\frac{z-\left|k_{2}\right|}{2\left|k_{1}\right|}+1\right)\left(\frac{z-\left|k_{2}\right|}{2\left|k_{1}\right|}+2\right) \cdots\left(\frac{z-\left|k_{2}\right|}{2\left|k_{1}\right|}+n\right)}{\frac{z+\left|k_{2}\right|}{2\left|k_{1}\right|}\left(\frac{z+\left|k_{2}\right|}{2\left|k_{1}\right|}+1\right) \cdots\left(\frac{z+\left|k_{2}\right|}{2\left|k_{1}\right|}+n\right)} .
$$

(6) $k_{1} k_{2}<0,\left|k_{2}\right| \geq 2, m+\left|k_{1}\right|=0$ and $\varphi(r)=C r^{\left|k_{2}\right|}$.

(7) $k_{1} k_{2}<0,\left|k_{2}\right|=1$ and $\widehat{\varphi}(z)=C \frac{\Gamma\left(\frac{z-1}{2\left|k_{1}\right|}\right) \Gamma\left(\frac{z+m+\left|k_{1}\right|+1}{2\left|k_{1}\right|}\right)}{\Gamma\left(\frac{z+2\left|k_{1}\right|+1}{2\left|k_{1}\right|}\right) \Gamma\left(\frac{z+m+k_{1} \mid-1}{2\left|k_{1}\right|}\right)}$.

In each condition (1)-(5), $\operatorname{rank}\left(\left[T_{e^{i k_{1} \theta} r^{m}}, T_{e^{i k_{2} \theta} \varphi}\right]\right)=0$. In each condition (6)-(7),

$$
\operatorname{Ran}\left(\left[T_{e^{i k_{1} \theta} r^{m}}, T_{e^{i k_{2} \theta} \varphi}\right]\right)=\operatorname{span}\left\{z^{\max \left\{k_{1}, k_{2}\right\}-1}\right\}
$$

and

$$
\left[T_{e^{i k_{1} \theta} r^{m}}, T_{e^{i k_{2} \theta} \varphi}\right]=C z^{\max \left\{k_{1}, k_{2}\right\}-1} \otimes z^{\max \left\{-k_{1},-k_{2}\right\}-1} .
$$

Therefore, $\operatorname{rank}\left(\left[T_{e^{i k_{1} \theta} r^{m}}, T_{e^{i k_{2} \theta} \varphi}\right]\right)=1$. 
Proof. Proceeding as in the proof of [15, Theorem 6], we can get that the commutator $\left[T_{e^{i k_{1} \theta} r^{m}}, T_{e^{i k_{2} \theta} \varphi}\right]$ on $L_{a}^{2}$ has finite rank if and only if $\left[T_{e^{i k_{1} \theta_{r}}}, T_{e^{i k_{2} \theta} \varphi}\right]\left(z^{n}\right)=0$ holds for any natural number $n \geq N_{1}$, or equivalently,

$$
\begin{aligned}
& 2\left(n+k_{2}+1\right) \widehat{r^{m}}\left(2 n+k_{1}+2 k_{2}+2\right) \widehat{\varphi}\left(2 n+k_{2}+2\right) \\
& =2\left(n+k_{1}+1\right) \widehat{r^{m}}\left(2 n+k_{1}+2\right) \widehat{\varphi}\left(2 n+2 k_{1}+k_{2}+2\right) .
\end{aligned}
$$

Notice that (6.1) is the same as (4.8), so from the proof of Theorem 4.4 we know that (6.1) holds if and only if one of the conditions (1)-(7) holds, as desired.

If one of the conditions (1)-(5) holds, then it is known that $\operatorname{rank}\left(\left[T_{e^{i k_{1} \theta} r^{m}}, T_{e^{i k_{2} \theta} \varphi}\right]\right)=0$. Now, we suppose condition (6) holds. Without loss of generality, we can assume that $k_{1}>0$, then $k_{1}=1$ and $N_{1}=-k_{2}$. Moreover, for any $0 \leq l<-k_{2}$, by Lemma 6.1 we get

$$
\begin{aligned}
{\left[T_{e^{i \theta} r^{-1}}, T_{e^{i k_{2} \theta} \varphi}\right]\left(z^{l}\right) } & = \begin{cases}0, & \text { if } 0 \leq l<-k_{2}-1, \\
-2\left(-k_{2}+1\right) \widehat{r^{-1}}\left(-2 k_{2}+1\right) 2 \widehat{\varphi}\left(-k_{2}+2\right), & \text { if } l=-k_{2}-1,\end{cases} \\
& = \begin{cases}0, & \text { if } 0 \leq l<-k_{2}-1, \\
\frac{1}{k_{2}}, & \text { if } l=-k_{2}-1 .\end{cases}
\end{aligned}
$$

Thus, $\operatorname{Ran}\left(\left[T_{e^{i \theta} r^{-1}}, T_{e^{i k_{2} \theta} \varphi}\right]\right)=\operatorname{span}\{1\}$ and $\left[T_{e^{i k_{1} \theta} r^{m}}, T_{e^{i k_{2} \theta} \varphi}\right]=-\left(1 \otimes z^{-k_{2}-1}\right)$. Next, we suppose condition (7) holds. We can also assume that $k_{1}>0$, then $k_{2}=-1$ and $N_{1}=1$. Moreover, by Lemma 6.1 we get

$$
\left[T_{e^{i k_{1} \theta} r^{m}}, T_{e^{-i \theta} \varphi}\right]\left(z^{0}\right)=-4 k_{1}\left(k_{1}+1\right) \widehat{r^{m}}\left(k_{1}+2\right) \widehat{\varphi}\left(2 k_{1}+1\right) z^{k_{1}-1} .
$$

Thus, $\operatorname{Ran}\left(\left[T_{e^{i \theta} r^{-1}}, T_{e^{i k_{2} \theta} \varphi}\right]\right)=\operatorname{span}\left\{z^{k_{1}-1}\right\}$ and

$$
\left[T_{e^{i k_{1} \theta} r^{m}}, T_{e^{-i \theta} \varphi}\right]=-4 k_{1}\left(k_{1}+1\right) \widehat{r^{m}}\left(k_{1}+2\right) \widehat{\varphi}\left(2 k_{1}+1\right) z^{k_{1}-1} \otimes 1 .
$$

This completes the proof.

We now observe several consequences of Theorem 6.2. First, we give two examples of the commutators on $L_{a}^{2}$ with nonzero finite rank, corresponding to the conditions (6) and (7) of Theorem 6.2.

Example 6.3. Let $\varphi(r)$ be a nonzero radial T-function on $D$, then on $L_{a}^{2}$, the following statements hold.

(1) The commutator $\left[T_{e^{2 i \theta} r^{6}}, T_{e^{-i \theta} \varphi(r)}\right]$ has finite rank if and only if $\varphi(r)=C\left(\frac{3}{r}-r^{3}\right)$. In this case, $\left[T_{e^{2 i \theta} r^{6}}, T_{e^{-i \theta} \varphi(r)}\right]=-\frac{3}{2} C(z \otimes 1)$.

(2) The commutator $\left[T_{e^{i \theta} r^{-1}}, T_{e^{-3 i \theta} \varphi(r)}\right]$ has finite rank if and only if $\varphi(r)=C r^{3}$. In this case, $\left[T_{e^{i \theta} r^{-1}}, T_{e^{-3 i \theta} \varphi(r)}\right]=-C\left(1 \otimes z^{2}\right)$.

Corollary 6.4. Let $k_{1}, k_{2} \in \mathbb{Z}$ such that $k_{1}>0$ or $k_{1}=-1$, and let $\varphi(r)$ be a nonzero radial T-function on $D$. Then on $L_{a}^{2}$, the following statements hold.

(a) The commutator $\left[T_{e^{i k_{1} \theta} r^{k_{1}}}, T_{e^{i k_{2} \theta} \varphi}\right]$ has finite rank if and only if

$$
k_{2}>-2 \text { and } \varphi(r)=C r^{k_{2}} .
$$

(b) The commutator $\left[T_{e^{-i k_{1} \theta} r^{k_{1}}}, T_{e^{i k_{2} \theta} \varphi}\right]$ has finite rank if and only if

$$
k_{2}<2 \text { and } \varphi(r)=C r^{-k_{2}} .
$$

Proof. If $k_{1}>0$, then by Theorem 6.2, the commutator $\left[T_{e^{i k_{1} \theta} r^{k_{1}}}, T_{e^{i k_{2} \theta} \varphi}\right]$ has finite rank if and only if $k_{2}$ satisfies one of the conditions (2), (4), (5) and (7) and

$$
\widehat{\varphi}(z)=\frac{C}{z+k_{2}} \text {. }
$$

In other words, $k_{2}>-2$ and $\varphi(r)=C r^{k_{2}}$. Similarly, if $k_{1}=-1$, then one of the conditions (2) and (4), (6) and (7) holds, and hence $k_{2}>-2$ and $\varphi(r)=C r^{k_{2}}$.

Combining condition (a) with the use of adjoint operators, one can get condition (b) holds. 
Corollary 6.5. Let $k_{1}, k_{2} \in \mathbb{Z}$ such that $k_{1} k_{2} \neq 0$, and let $m_{1}, m_{2} \in \mathbb{R}$ such that greater than or equal to -1. Then the commutator $\left[T_{e^{i k_{1} \theta} r^{m_{1}}}, T_{e^{i k_{2} \theta} r^{m_{2}}}\right]$ has finite rank on $L_{a}^{2}$ if and only if one of the following conditions holds:

(1) $k_{1}=k_{2}$ and $m_{1}=m_{2}$.

(2) $k_{1}=m_{1}$ and $k_{2}=m_{2}$.

(3) $k_{1}=-m_{1}$ and $k_{2}=-m_{2}$.

Proof. The proof is similar to Corollary 4.7

Next, we will discuss the finite rank generalized semicommutators of quasihomogeneous Toeplitz operators on $L_{a}^{2}$. First, we give the following result.

Proposition 6.6. Let $k_{1}, k_{2} \in \mathbb{Z}$, and let $m \in \mathbb{R}, m \geq-1$. Then for radial T-functions $\varphi$ and $\psi$ on $D$, the generalized semicommutator $T_{e^{i k_{1} \theta} r_{m}} T_{e^{i k_{2} \theta} \varphi}-T_{e^{i\left(k_{1}+k_{2}\right) \theta} \psi}$ has finite rank on $L_{a}^{2}$ if and only if

$$
\psi(r)=\frac{\varphi(r)}{r^{k_{1}}}-\left(m+k_{1}\right) r^{m+k_{2}} \int_{r}^{1} \frac{\varphi(t)}{t^{m+k_{1}+k_{2}+1}} d t
$$

Proof. Using the same argument as in the proof of Proposition 4.1, we see that $T_{e^{i k_{1} \theta} r^{m}} T_{e^{i k_{2} \theta} \varphi}-$ $T_{e^{i\left(k_{1}+k_{2}\right) \theta} \psi}$ on $L_{a}^{2}$ has finite rank if and only if

$$
2\left(n+k_{2}+1\right) \widehat{r^{m}}\left(2 n+k_{1}+2 k_{2}+2\right) \widehat{\varphi}\left(2 n+k_{2}+2\right)=\widehat{\psi}\left(2 n+k_{1}+k_{2}+2\right)
$$

holds for any natural number $n \geq N_{2}$, and hence

$$
\left(r^{m+k_{1}+2 k_{2}+2 N_{2}}\right) *_{M}\left(r^{k_{2}+2 N_{2}} \varphi\right)=\left(r^{2 k_{2}+2 N_{2}}\right) *_{M}\left(r^{k_{1}+k_{2}+2 N_{2}} \psi\right),
$$

which is equivalent to

$$
r^{m+k_{1}} \int_{r}^{1} \frac{\varphi(t)}{t^{m+k_{1}+k_{2}+1}} d t=\int_{r}^{1} \frac{\psi(t)}{t^{k_{2}-k_{1}+1}} d t .
$$

By differentiating both sides, we get (6.2) holds.

Remark 6.7. Let $k_{1}, k_{2}, m$ and $\varphi$ be as in Proposition 6.6, and let $\psi$ be defined by (6.2). If $\frac{\varphi(r)}{r^{k_{1}}}$ is a T-function and either $m+k_{2} \geq-1$ or $m+k_{1}=0$ hold, then $\psi$ is a T-function, and hence $T_{e^{i k_{1} \theta} r^{m}} T_{e^{i k_{2} \theta} \varphi}-T_{e^{i\left(k_{1}+k_{2}\right) \theta} \psi}$ has finite rank. In fact, since $\frac{\varphi(r)}{r^{k_{1}}}$ is a T-function, the desired result is obvious when $m+k_{1}=0$. Now we suppose $m+k_{2} \geq-1$. Then we have

$$
\begin{aligned}
\int_{0}^{1}\left|r^{m+k_{2}} \int_{r}^{1} \frac{\varphi(t)}{t^{m+k_{1}+k_{2}+1}} d t\right| r d r & \leq \int_{0}^{1} r^{m+k_{2}+1} d r \int_{r}^{1} \frac{|\varphi(t)|}{t^{m+k_{1}+k_{2}+1}} d t \\
& =\int_{0}^{1} \frac{|\varphi(t)|}{t^{m+k_{1}+k_{2}+1}} d t \int_{0}^{t} r^{m+k_{2}+1} d r \\
& =\frac{1}{m+k_{2}+2}\left\|\frac{\varphi(r)}{r^{k_{1}}}\right\|_{L^{1}}<\infty .
\end{aligned}
$$

Moreover,

$$
\left|r^{m+k_{2}} \int_{r}^{1} \frac{\varphi(t)}{t^{m+k_{1}+k_{2}+1}} d t\right| \leq \int_{r}^{1}\left|\frac{\varphi(t)}{t^{k_{1}}}\right| \frac{d t}{t} \leq \frac{1}{r^{2}}\left\|\frac{\varphi(r)}{r^{k_{1}}}\right\|_{L^{1}} .
$$

Therefore, the radial function $r^{m+k_{2}} \int_{r}^{1} \frac{\varphi(t)}{t^{m+k_{1}+k_{2}+1}} d t$ is "nearly bounded" on $D$, and hence $\psi$ is a T-function.

The following theorem completely characterizes the finite rank generalized semicommutators of two Toeplitz operators on $L_{a}^{2}$ with monomial symbols.

Theorem 6.8. Let $k_{1}, k_{2} \in \mathbb{Z}$, and let $m_{1}, m_{2} \geq-1$. Then for a radial $T$-function $\psi$ on $D$, the generalized semicommutator $T_{e^{i k_{1} \theta} r^{m_{1}}} T_{e^{i k_{2} \theta} r^{m_{2}}}-T_{e^{i\left(k_{1}+k_{2}\right) \theta} \psi}$ has finite rank on $L_{a}^{2}$ if and only if

$$
\psi(r)= \begin{cases}\frac{k_{1}+m_{1}}{m_{1}+k_{2}-m_{2}+k_{1}} r^{m_{1}+k_{2}}-\frac{m_{2}-k_{2}}{m_{1}+k_{2}-m_{2}+k_{1}} r^{m_{2}-k_{1}}, & \text { if } m_{1}+k_{2} \neq m_{2}-k_{1}, \\ r^{m_{1}+k_{2}}\left[1+\left(m_{1}+k_{1}\right) \log r\right], & \text { if } m_{1}+k_{2}=m_{2}-k_{1},\end{cases}
$$


and one of the following conditions holds:

(1) $k_{1}+m_{1} \neq 0, k_{2}-m_{2} \neq 0, m_{1}+k_{2} \geq-1$ and $m_{2}-k_{1} \geq-1$.

(2) $k_{1}+m_{1}=0$ and $m_{2}-k_{1} \geq-1$.

(3) $k_{2}-m_{2}=0$ and $m_{1}+k_{2} \geq-1$.

Furthermore, $T_{e^{i k_{1} \theta} r^{m_{1}}} T_{e^{i k_{2} \theta} r^{m_{2}}}-T_{e^{i\left(k_{1}+k_{2}\right) \theta} \psi}$ on $L_{a}^{2}$ has nonzero finite rank if and only if (6.3) holds and one of the following conditions holds:

- $k_{1}+k_{2} \geq 0, k_{2} \leq-2$ and condition (1) holds. In this case,

$$
\operatorname{Ran}\left(T_{e^{i k_{1} \theta} r^{m_{1}}} T_{e^{i k_{2} \theta} r^{m_{2}}}-T_{e^{i\left(k_{1}+k_{2}\right) \theta} \psi}\right)=\operatorname{span}\left\{z^{k_{1}+k_{2}}, \cdots, z^{k_{1}-2}\right\}
$$

and

$$
T_{e^{i k_{1} \theta} r^{m_{1}}} T_{e^{i k_{2} \theta} r^{m_{2}}}-T_{e^{i\left(k_{1}+k_{2}\right) \theta} \psi}=\sum_{j=0}^{-k_{2}-2} C_{j}\left(z^{j+k_{1}+k_{2}} \otimes z^{j}\right)
$$

for nonzero constant $C_{j}$. Therefore, $\operatorname{rank}\left(T_{e^{i k_{1} \theta} r^{m_{1}}} T_{e^{i k_{2} \theta} r^{m_{2}}}-T_{e^{i\left(k_{1}+k_{2}\right) \theta} \psi}\right)=-k_{2}-1$.

- $k_{1}+k_{2} \geq 0, k_{2}=-1$ and one of the conditions (2)-(3) holds. In this case,

$$
\operatorname{Ran}\left(T_{e^{i k_{1} \theta}{ }_{r} m_{1}} T_{e^{i k_{2} \theta} r^{m_{2}}}-T_{e^{i\left(k_{1}+k_{2}\right) \theta} \psi}\right)=\operatorname{span}\left\{z^{k_{1}-1}\right\}
$$

and

$$
T_{e^{i k_{1} \theta} r^{m_{1}}} T_{e^{i k_{2} \theta} r^{m_{2}}}-T_{e^{i\left(k_{1}+k_{2}\right) \theta} \psi}=C\left(z^{k_{1}-1} \otimes 1\right)
$$

for nonzero constant $C$. Therefore, $\operatorname{rank}\left(T_{e^{i k_{1} \theta} r^{m_{1}}} T_{e^{i k_{2} \theta} r^{m_{2}}}-T_{e^{i\left(k_{1}+k_{2}\right) \theta} \psi}\right)=1$.

- $k_{1}+k_{2}<0, k_{1} \geq 2$ and condition (1) holds. In this case,

$$
\operatorname{Ran}\left(T_{e^{i k_{1} \theta} r^{m_{1}}} T_{e^{i k_{2} \theta} r^{m_{2}}}-T_{e^{i\left(k_{1}+k_{2}\right) \theta} \psi}\right)=\operatorname{span}\left\{1, \cdots, z^{k_{1}-2}\right\}
$$

and

$$
T_{e^{i k_{1} \theta} r^{m_{1}}} T_{e^{i k_{2} \theta} r^{m_{2}}}-T_{e^{i\left(k_{1}+k_{2}\right) \theta} \psi}=\sum_{j=0}^{k_{1}-2} C_{j}\left(z^{j} \otimes z^{j-k_{1}-k_{2}}\right)
$$

for nonzero constant $C_{j}$. Therefore, rank $\left(T_{e^{i k_{1} \theta} r^{m_{1}}} T_{e^{i k_{2} \theta} r^{m_{2}}}-T_{e^{i\left(k_{1}+k_{2}\right) \theta} \psi}\right)=k_{1}-1$.

- $k_{1}+k_{2}<0, k_{1}=1$ and condition (2) holds. In this case,

$$
\operatorname{Ran}\left(T_{e^{i k_{1} \theta} r^{m_{1}}} T_{e^{i k_{2} \theta} r^{m_{2}}}-T_{e^{i\left(k_{1}+k_{2}\right) \theta} \psi}\right)=\operatorname{span}\{1\}
$$

and

$$
T_{e^{i k_{1} \theta} r^{m_{1}}} T_{e^{i k_{2} \theta} r^{m_{2}}}-T_{e^{i\left(k_{1}+k_{2}\right) \theta} \psi}=C\left(1 \otimes z^{-k_{1}-k_{2}}\right)
$$

for nonzero constant $C$. Therefore, $\operatorname{rank}\left(T_{e^{i k_{1} \theta} r^{m_{1}}} T_{e^{i k_{2} \theta} r^{m_{2}}}-T_{e^{i\left(k_{1}+k_{2}\right) \theta} \psi}\right)=k_{1}$.

Proof. By Proposition 6.6. $T_{e^{i k_{1} \theta} r^{m_{1}}} T_{e^{i k_{2} \theta} r^{m_{2}}}-T_{e^{i\left(k_{1}+k_{2}\right) \theta} \psi}$ has finite rank if and only if

$$
\begin{aligned}
\psi(r) & =\frac{r^{m_{2}}}{r^{k_{1}}}-\left(m_{1}+k_{1}\right) r^{m_{1}+k_{2}} \int_{r}^{1} \frac{t^{m_{2}}}{t^{m_{1}+k_{1}+k_{2}+1}} d t \\
& = \begin{cases}\frac{k_{1}+m_{1}}{m_{1}+k_{2}-m_{2}+k_{1}} r^{m_{1}+k_{2}}-\frac{m_{2}-k_{2}}{m_{1}+k_{2}-m_{2}+k_{1}} r^{m_{2}-k_{1}}, & \text { if } m_{1}+k_{2} \neq m_{2}-k_{1}, \\
r^{m_{1}+k_{2}}\left[1+\left(m_{1}+k_{1}\right) \log r\right], & \text { if } m_{1}+k_{2}=m_{2}-k_{1},\end{cases}
\end{aligned}
$$

and $\psi(r)$ is a T-function. It is also clear that $\psi(r)$ defined by (6.3) is a T-function if and only if one of the conditions (1)-(3) holds.

Next, we need to determine the range of the generalized semicommutator $T_{e^{i k_{1} \theta} r^{m_{1}}} T_{e^{i k_{2} \theta} r^{m_{2}}}-$ $T_{e^{i\left(k_{1}+k_{2}\right) \theta} \psi}$. We have shown in Proposition 6.6 that $\left(T_{e^{i k_{1} \theta} r^{m_{1}}} T_{e^{i k_{2} \theta} r^{m_{2}}}-T_{e^{i\left(k_{1}+k_{2}\right) \theta} \psi}\right)\left(z^{n}\right)=0$ holds for any natural number $n \geq N_{2}$.

Now, we assume that $k_{1}+k_{2} \geq 0$. If $k_{2} \geq 0$, then $N_{2}=0$, and hence

$$
\operatorname{rank}\left(T_{e^{i k_{1} \theta} r^{m_{1}}} T_{e^{i k_{2} \theta} r^{m_{2}}}-T_{e^{i\left(k_{1}+k_{2}\right) \theta} \psi}\right)=0 .
$$

If $k_{2} \leq-1$, then $N_{2}=-k_{2}$. For any $0 \leq n<-k_{2}$, by Lemma 6.1 we get

$$
\left[T_{e^{i k_{1} \theta} r^{m_{1}}} T_{e^{i k_{2} \theta} r^{m_{2}}}-T_{e^{i\left(k_{1}+k_{2}\right) \theta} \psi}\right]\left(z^{n}\right)=2\left(n+k_{1}+k_{2}+1\right) \widehat{\psi}\left(2 n+k_{1}+k_{2}+2\right) z^{n+k_{1}+k_{2}} .
$$


On the other hand, (6.3) implies that

$$
\widehat{\psi}(z)= \begin{cases}\frac{1}{m_{1}+k_{2}-m_{2}+k_{1}}\left(\frac{k_{1}+m_{1}}{m_{1}+k_{2}+z}-\frac{m_{2}-k_{2}}{m_{2}-k_{1}+z}\right), & \text { if } m_{1}+k_{2} \neq m_{2}-k_{1}, \\ \frac{z-k_{1}+k_{2}}{\left(m_{1}+k_{2}+z\right)^{2}}, & \text { if } m_{1}+k_{2}=m_{2}-k_{1},\end{cases}
$$

which is well defined on $\{z: \operatorname{Re} z \geq 2\}$. Then a direct calculation shows that

$$
\widehat{\psi}\left(2 n+k_{1}+k_{2}+2\right)=0 \Longleftrightarrow n=-k_{2}-1, k_{1}+m_{1} \neq 0 \text { and } k_{2}-m_{2} \neq 0 .
$$

Thus, if condition (1) holds, then

$$
\operatorname{rank}\left(T_{e^{i k_{1} \theta} r^{m_{1}}} T_{e^{i k_{2} \theta} r^{m_{2}}}-T_{e^{i\left(k_{1}+k_{2}\right) \theta} \psi}\right)=\max \left\{0,-k_{2}-1\right\},
$$

and if either condition (2) or condition (3) holds, then

$$
\operatorname{rank}\left(T_{e^{i k_{1} \theta} r^{m_{1}}} T_{e^{i k_{2} \theta} r^{m_{2}}}-T_{e^{i\left(k_{1}+k_{2}\right) \theta} \psi}\right)=-k_{2} .
$$

Since $-k_{1} \leq k_{2} \leq-1$ and $m_{1}, m_{2} \geq-1$, then both condition (2) and condition (3) imply that $k_{2}=-1$, as desired.

If $k_{1}+k_{2}<0$, then by using the adjoint operator, the desired results are obvious. This completes the proof.

Below we present the examples of nonzero generalized semicommutators of finite rank on $L_{a}^{2}$.

Example 6.9. Let $\psi$ be a radial T-function on $D$, then on $L_{a}^{2}$, the following statements hold.

(1) $T_{e^{2 i \theta} r} T_{e^{-2 i \theta} r^{2}}-T_{\psi}$ has finite rank if and only if $\psi(r)=4-3 r^{-1}$.

In this case, $T_{e^{2 i \theta} r} T_{e^{-2 i \theta} r^{2}}-T_{\psi}=2(1 \otimes 1)$.

(2) $T_{e^{3 i \theta} r^{5}} T_{e^{-i \theta} r^{-1}}-T_{e^{2 i \theta} \psi}$ has finite rank if and only if $\psi(r)=r^{4}$.

In this case, $T_{e^{3 i \theta} r^{5}} T_{e^{-i \theta} r^{-1}}-T_{e^{2 i \theta} \psi}=-\frac{3}{4}\left(z^{2} \otimes 1\right)$.

(3) $T_{e^{3 i \theta} r^{5}} T_{e^{-4 i \theta} r^{3}}-T_{e^{-i \theta} \psi}$ has finite rank if and only if $\psi(r)=8 r-7$.

In this case, $T_{e^{4 i \theta} r^{3}} T_{e^{-3 i \theta} r^{5}}-T_{e^{i \theta} \psi}=\frac{4}{3}(1 \otimes z)+\frac{4}{5}\left(z \otimes z^{2}\right)$.

(4) $T_{e^{i \theta} r^{-1}} T_{e^{-3 i \theta} r^{3}}-T_{e^{-2 i \theta} \psi}$ has finite rank if and only if $\psi(r)=r^{2}$.

In this case, $T_{e^{i \theta} r^{-1}} T_{e^{-3 i \theta} r^{3}}-T_{e^{-2 i \theta} \psi}=-\left(1 \otimes z^{2}\right)$.

\section{Further RESUlts AND REMARKS}

There is a general feeling that the restrictions on their symbols for Toeplitz operators to satisfy certain algebraic properties on $L_{h}^{2}$ are more than $L_{a}^{2}$, as the orthonormal basis for $L_{h}^{2}$ involves co-analytic monomial. We list here some results which illustrate this view.

(1). Choe and Lee [11] showed that two analytic Toeplitz operators on $L_{h}^{2}$ commute only when their symbols and the constant function 1 are linearly dependent, but analytic Toeplitz operators always commute on $L_{a}^{2}$.

(2). Guo and Zheng 24 obtain a criterion for compactness of Toeplitz operators with bounded symbols on $L_{h}^{2}$, which requires one more condition than that on $L_{a}^{2}$.

(3). We showed in 22 that Toeplitz operators with bounded symbols on $L_{h}^{2}$ commute with $T_{e^{i k \theta} r^{m}}$ only in trivial cases, but [16] showed there are many nontrivial Toeplitz operators commuting with $T_{e^{i k \theta} r^{m}}$ on $L_{a}^{2}$.

(4). We proved in 21 that the product of two Toeplitz operators with bounded symbols $T_{e^{i k_{1} \theta} \varphi} T_{e^{i k_{2} \theta} r^{m}}$ on $L_{h}^{2}$ is a Toeplitz operator only in trivial cases, but it also holds on $L_{a}^{2}$ when either $\overline{e^{i k_{1} \theta} \varphi}$ or $e^{i k_{2} \theta} r^{m}$ is analytic.

However, comparing to the Bergman space case, the theory of Toeplitz operators on $L_{h}^{2}$ has its own unique properties. The most unexpected results to us are the symmetry properties obtained in [9, 20, 21]. In the following, we will show the relation between finite rank commutators (or generalized semicommutators) of quasihomogenous Toeplitz operators on $L_{h}^{2}$ and that on $L_{a}^{2}$.

Proposition 7.1. Let $k_{1}, k_{2} \in \mathbb{Z}$, and let $\varphi_{1}, \varphi_{2}$ and $\psi$ be radial T-functions. Then the following statements hold.

(1) The commutator $\left[T_{e^{i k_{1} \theta} \varphi_{1}}, T_{e^{i k_{2} \theta} \varphi_{2}}\right]$ has finite rank on $L_{h}^{2}$ if and only if $\left[T_{e^{i k_{1} \theta} \varphi_{1}}, T_{e^{i k_{2} \theta} \varphi_{2}}\right]$ has finite rank on $L_{a}^{2}$. 
(2) If the generalized semicommutator $T_{e^{i k_{1} \theta} \varphi_{1}} T_{e^{i k_{2} \theta} \varphi_{2}}-T_{e^{i\left(k_{1}+k_{2}\right) \theta} \psi}$ has finite rank on $L_{h}^{2}$, then both $T_{e^{i k_{1} \theta} \varphi_{1}} T_{e^{i k_{2} \theta} \varphi_{2}}-T_{e^{i\left(k_{1}+k_{2}\right) \theta} \psi}$ and $T_{e^{i k_{2} \theta} \varphi_{2}} T_{e^{i k_{1} \theta} \varphi_{1}}-T_{e^{i\left(k_{1}+k_{2}\right) \theta} \psi}$ have finite rank on $L_{a}^{2}$.

Proof. The same reasoning as in the proof of [15, Theorem 6] shows that $\left[T_{e^{i k_{1} \theta} \varphi_{1}}, T_{e^{i k_{2} \theta} \varphi_{2}}\right]$ has finite rank on $L_{a}^{2}$ if and only if (4.1) holds for any natural number $n \geq N_{1}$. Then it follows from Proposition 4.1 that condition (1) holds.

Now, we suppose $T_{e^{i k_{1} \theta} \varphi_{1}} T_{e^{i k_{2} \theta} \varphi_{2}}-T_{e^{i\left(k_{1}+k_{2}\right) \theta} \psi}$ has finite rank on $L_{h}^{2}$. Then by Proposition 5.1 we get (5.1) holds for any natural number $n \geq N_{2}$ and (5.2) holds for any natural number $n \geq N_{3}$. On the other hand, the same reasoning as in the proof of [15, Theorem 4] shows that $T_{e^{i k_{1} \theta} \varphi_{1}} T_{e^{i k_{2} \theta} \varphi_{2}}-T_{e^{i\left(k_{1}+k_{2}\right) \theta} \psi}$ has finite rank on $L_{a}^{2}$ if and only if (5.1) holds for $n \geq N_{2}$, and $T_{e^{i k_{2} \theta} \varphi_{2}} T_{e^{i k_{1} \theta} \varphi_{1}}-T_{e^{i\left(k_{1}+k_{2}\right) \theta} \psi}$ has finite rank on $L_{a}^{2}$ if and only if

$$
2\left(n+k_{1}+1\right) \widehat{\varphi_{1}}\left(2 n+k_{1}+2\right) \widehat{\varphi_{2}}\left(2 n+2 k_{1}+k_{2}+2\right)=\widehat{\psi}\left(2 n+k_{1}+k_{2}+2\right)
$$

holds for any natural number $n \geq \max \left\{0,-k_{1},-k_{1}-k_{2}\right\}$. Replacing $n$ by $n-k_{1}-k_{2}$, we see that the above equation is the same as (5.2). This completes the proof.

Remark 7.2. (1) Suppose $k_{1}, k_{2}, m, \varphi$ and $\psi$ satisfy one of the conditions in Theorem 5.4. Then as a direct consequence of Proposition 7.1, we get that $T_{e^{i k_{1} \theta} r^{m}} T_{e^{i k_{2} \theta} \varphi}-T_{e^{i\left(k_{1}+k_{2}\right) \theta} \psi}$ has finite rank on $L_{a}^{2}$.

(2) In general, the rank of finite rank commutator or generalized semicommutator of quasihomogenous Toeplitz operators on $L_{h}^{2}$ should be greater than or equal to that on $L_{a}^{2}$. However, it is not all the cases. For example, we have shown in Theorem 4.4 and Theorem 5.4 that if $m \in \mathbb{R}, m \geq-1$ and $\varphi(r)=\frac{m+1}{2} r^{-1}-\frac{m-1}{2} r$, then on $L_{h}^{2}$,

$$
T_{e^{i \theta} r^{m}} T_{e^{-i \theta} \varphi}=T_{e^{-i \theta} \varphi} T_{e^{i \theta} r^{m}}
$$

and

$$
T_{e^{i \theta} r^{m}} T_{e^{-i \theta} \varphi}=T_{1} .
$$

However, by a direct calculation, one can get that on $L_{a}^{2}$,

$$
\left[T_{e^{i \theta} r^{m}}, T_{e^{-i \theta} \varphi}\right]=-\left(T_{e^{i \theta} r_{m}} T_{e^{-i \theta} \varphi}-T_{1}\right)=1 \otimes 1 .
$$

Therefore, both $\left[T_{e^{i \theta} r^{m}}, T_{e^{-i \theta} \varphi}\right]$ and $T_{e^{i \theta} r^{m}} T_{e^{-i \theta} \varphi}-T_{1}$ have rank zero on $L_{h}^{2}$, and rank one on $L_{a}^{2}$.

Acknowledgements: This work was supported in part by the National Natural Science Foundation of China (Grant Nos. 11201331; 11371276; 11401431).

\section{REFERENCES}

[1] P. Ahern and Ž. Čučković, A theorem of Brown-Halmos type for Bergman space Toeplitz operators, J. Funct. Anal. 187 (2001), 200-210.

[2] S. Axler and Ž. Cučković, Commuting Toeplitz operators with harmonic symbols, Integral Equations Operator Theory. 14 (1991), 1-12.

[3] M. Abramowitz and I. A. Stegun (Eds), Handbook of Mathematical Functions with Formulas, Graphs, and Mathematical Tables, National Bureau of Standards, Applied Mathematics Series 55, 4th printing, with corrections, Washington, 1965.

[4] S. Axler, S. Chang and D. Sarason, Product of Toeplitz operators, Integral Equations Operator Theory. 1 (1978), 285-309.

[5] A. Brown and P.R. Halmos, Algebraic properties of Toeplitz operators, J. Reine Angew. Math. 213 (1964), 89-102.

[6] W. Bauer and H. Issa, Commuting Toeplitz operators with quasi-homogeneous symbols on the SegalBargmann space, J. Math. Anal. Appl. 386 (2012), 213-235.

[7] W. Bauer and Y.J. Lee, Commuting Toeplitz operators on the Segal-Bargmann space, J. Funct. Anal. 260 (2011), 460-489.

[8] W. Bauer and N. Vasilevski, Commutative Toeplitz Banach Algebras on the Ball and Quasi-Nilpotent Group Action, Integr. Equ. Oper. Theory. 72 (2012), 223-240.

[9] Y. Chen, H. Koo and Y.J. Lee, Ranks of commutators of Toeplitz operators on the harmonic Bergman space, Integral Equations Operator Theory. 75 (2013), 31-38. 
[10] B.R. Choe, H. Koo, Y.J. Lee, Commuting Toeplitz operators on the polydisk. Trans. Am. Math. Soc. 356 (2004), 1727-1749.

[11] B.R. Choe and Y.J. Lee, Commuting Toeplitz operators on the harmonic Bergman spaces, Michigan Math. J. 46 (1999), 163-174.

[12] B.R. Choe and Y.J. Lee, Commutants of analytic Toeplitz operators on the harmonic Bergman space, Integral Equations Operator Theory. 50 (2004), 559-564.

[13] Ž. Čučković, Finite rank perturbations of Toeplitz operators, Integral Equations Operator Theory. 59 (2007), 345-353.

[14] Ž. Čučković and T. Le, Toeplitz operators on Bergman spaces of polyanalytic functions, Bull. London Math. Soc. 44 (2012), 961-973.

[15] Ž. Čučković and I. Louhichi, Finite rank commutators and semicommutators of quasihomogeneous Toeplitz operators, Complex Anal. Oper. Theory. 2 (2008), 429-439.

[16] Ž. Čučković and N.V. Rao, Mellin transform, monomial symbols, and commuting Toeplitz operators, J. Funct. Anal. 154 (1998), 195-214.

[17] X. Ding and D. Zheng, Finite rank commutator of Toeplitz operators or Hankel operators, Houston J. Math. 34 (2008), 1099-1119.

[18] X.T. Dong and Z.H. Zhou, Algebraic properties of Toeplitz operators with separately quasihomogeneous symbols on the Bergman space of the unit ball, J. Operator Theory. 66 (2011), 193-207.

[19] X.T. Dong and Z.H. Zhou, Products of Toeplitz operators on the harmonic Bergman space, Proc. Amer. Math. Soc. 138 (2010),1765-1773.

[20] X.T. Dong and Z.H. Zhou, Commuting quasihomogeneous Toeplitz Operators on the Harmonic Bergman Space, Complex Anal. Oper. Theory. 7 (2013), 1267-1285.

[21] X.T. Dong and Z.H. Zhou, Product equivalence of quasihomogeneous Toeplitz Operators on the Harmonic Bergman Space, Studia Math. 219 (2013), 163-175.

[22] X.T. Dong, C. Liu and Z.H. Zhou, Quasihomogeneous Toeplitz Operators with integrable symbols on the Harmonic Bergman Space, Bull. Austral. Math. Soc. 90 (2014), 494-503.

[23] K. Guo, S. Sun and D. Zheng, Finite rank commutators and semicommutators of Toeplitz operators with harmonic symbols. Ill. J. Math. 51 (2007), 583-596.

[24] K. Guo, D. Zheng, Toeplitz algebra and Hankel algebra on the harmonic Bergman space, J. Math. Anal. Appl. 276 (2002) 213-230.

[25] I. Louhichi, E. Strouse and L. Zakariasy, Products of Toeplitz operators on the Bergman space, Integral Equations Operator Theory. 54 (2006), 525-539.

[26] I. Louhichi and L. Zakariasy, On Toeplitz operators with quasihomogeneous symbols, Arch. Math. 85 (2005), $248-257$.

[27] I. Louhichi and L. Zakariasy, Quasihomogeneous Toeplitz operators on the harmonic Bergman space, Arch. Math. 98 (2012), 49-60.

[28] R. Quiroga-Barranco and A. Sanchez-nungaray, Toeplitz Operators with Quasi-radial quasi-homogeneous symbols and bounles of lagrangian frames, J. Oper. Theory. 71 (2014), 199-222.

[29] R. Quiroga-Barranco and A. Sanchez-nungaray, Toeplitz Operators with Quasi-Homogeneuos Quasi-Radial Symbols on some Weakly Pseudoconvex Domains, Complex Anal. Oper. Theory, 9 (2015), 1111-1134.

[30] N. Vasilevski, Quasi-radial quasi-homogeneous symbols and commutative Banach algebras of Toeplitz operators, Integral Equations Operator Theory. 66 (2010), 141-152.

[31] D. Zheng, Commuting Toeplitz Operators with Pluriharmonic Symbols, Trans. Amer. Math. Soc. 350 (1998), 1595-1618.

[32] Z.H. Zhou and X.T. Dong, Algebraic properties of Toeplitz operators with radial symbols on the Bergman space of the unit ball, Integral Equations Operator Theory. 64 (2009), 137-154.

Xing-Tang Dong (Corresponding author)

Department of Mathematics, Tianjin University, Tianjin 300354, P.R. China. E-mail address: dongxingtang@163.com

Ze-Hua Zhou

Department of Mathematics, Tianjin University, Tianjin 300354, P.R. China.

E-mail address: zehuazhoumath@aliyun.com; zhzhou@tju.edu.cn 